\title{
EfFicient Conditional Proxy Re- ENCRYPTION WITH CHOSEN CIPHER TEXT SECURITY
}

\author{
S. Sree Vivek ${ }^{1}$, S. Sharmila Deva Selvi ${ }^{1}$, V. Radhakishan ${ }^{2}$, C. Pandu Rangan ${ }^{1}$ \\ ${ }^{1}$ Department of Computer Science and Engineering, Indian Institute of Technology Madras \\ svivek@cse.iitm.ac.in, sharmila@cse.iitm.ac.in, prangandiitm.ac.in \\ ${ }^{2}$ National Institute of Technology Trichy, India \\ vrkishan@gmail.com
}

\begin{abstract}
In a proxy re-encryption (PRE) scheme, Alice gives a special information to a proxy that allows it to transform messages encrypted under Alice's public key into a encryption under Bob's public key such that the message is not revealed to the proxy. In [14], Jian Weng and others introduced the notion of conditional proxy re-encryption (C-PRE) with bilinear pairings. Later, a break for the same was published in [17] and a new C-PRE scheme with bilinear pairings was introduced. In C-PRE, the proxy also needs to have the right condition key to transform the ciphertext (associated with a condition set by Alice) under Alice's public key into ciphertext under Bob's public key, so that Bob can decrypt it. In this paper, we propose an efficient C-PRE scheme which uses substantially less number of bilinear pairings when compared to the existing one [17]. We then prove its chosen-ciphertext security under modified Computational Diffie-Hellman $(\mathrm{mCDH})$ and modified Computational Bilinear Diffie-Hellman $(m C B D H)$ assumptions in the random oracle model.
\end{abstract}

\section{KEYWORDS}

Random Oracle Model, Proxy Re-Cryptography, Conditional Proxy Re-encryption, Chosen Ciphertext Security.

\section{INTRODUCTION}

Encryption is used as a building block of any application requiring confidentiality. Let $\mathrm{pk}_{\mathrm{i}}$ and $\mathrm{pk}_{\mathrm{j}}$ be two independent public keys. As pointed out by Mambo and Okamato in [15], it is a common situation in practice where a data encrypted under $\mathrm{pk}_{\mathrm{i}}$ is required to be encrypted under $p k_{j}(j \neq i)$. When the holder of $s k_{i}$ is online, $E_{i}(m)$ is decrypted using $s k_{i}$ and then message $m$ is encrypted under $\mathrm{pk}_{\mathrm{j}}$ giving $\mathrm{E}_{\mathrm{j}}(\mathrm{m})$. But in many applications like encrypted mail forwarding, secure distributed file systems, and outsourced filtering of encrypted spam, when the holder of $\mathrm{sk}_{\mathrm{i}}$ is not online, this has to be done by an untrusted party.

In 1998 Blaze, Bleumar, and Strauss [9] introduced the concept of proxy re-encryption (PRE). A re-encryption key $\left(\mathrm{rk}_{\mathrm{i}, \mathrm{j}}\right)$ is given to a potentially untrusted proxy so that the proxy can transform a message $m$ encrypted under public key $\mathrm{pk}_{\mathrm{i}}$ into an encryption of the same message $m$ under a different public key $\mathrm{pk}_{\mathrm{j}}$ without knowing the message. A PRE scheme can be of two types - unidirectional and bidirectional. The former is a scheme in which a re-encryption key $\left(\mathrm{rk}_{\mathrm{i} \rightarrow \mathrm{j}}\right)$ can be used to transform from $\mathrm{pk}_{\mathrm{i}}$ to $\mathrm{pk}_{\mathrm{j}}$ but not vice versa and the latter is a scheme in which the same re-encryption key $\left(\mathrm{rk}_{\mathrm{i} \leftrightarrow \mathrm{j}}\right)$ can be used to transform from $\mathrm{pk}_{\mathrm{i}}$ to $\mathrm{pk}_{\mathrm{j}}$ and vice versa. The re-encryption algorithm can be of two types - single hop, in which the re-encrypted ciphertext cannot be further re-encrypted and multi hop, in which the re-encrypted ciphertext can be further re-encrypted. 
International Journal of Network Security \& Its Applications (IJNSA), Vol.4, No.2, March 2012

PRE can be used in many applications, including simplification of key distribution [9], key escrow [13], multicast [19], distributed file systems [3, 5], security in publish/subscribe systems [4], secure certified email mailing lists [20, 23], the DRM of Apple's iTunes [22], interoperable architecture of DRM [21], access control [11], and privacy for public transportation [7]. Hohenberger and others published a result of securely obfuscating re-encryption [16], which is the first positive result for obfuscating an encryption functionality. Shao and Cao have proposed a unidirectional PRE scheme without pairing [2]. Matthew Green and Giuseppe Ateniese have proposed a PRE scheme for ID-based cryptosystems [18].

Ran Canetti and Susan Hohenberger proposed a definition of security against chosen-ciphertext attacks for PRE schemes and presented a scheme that satisfied the definition [1]. In 2009, Jian Weng and others [14] introduced the concept of C-PRE, whereby Alice has a fine-grained control over the delegation. As a result, Alice can flexibly assign Bob the decryption capability based on the conditions attached to the messages using a proxy. For example, suppose Alice is on a vacation. She can make Bob to read only those messages which have the keyword "urgent" in their subject. This flexible delegation is obviously not possible with PRE schemes. In this paper, two separate keys are used - a partial re-encryption key and a condition key. The message can be delegated by the proxy only if both the keys are known.

Later in 2009, Jian Weng and others published a break of the scheme in [14] and gave a new scheme for C-PRE [17], which combines the re-encryption key and the condition key into a single key, which is then used for re-encryption. Also Cheng-Kang Chu and others in [8] introduced a generalized version of C-PRE named conditional proxy broadcast re-encryption (CPBRE), in which the proxy can re-encrypt the ciphertexts for a set of users at a time.

In this paper, we propose an efficient C-PRE scheme (single-hop and unidirectional) which uses significantly less number of bilinear pairings when compared to the existing schemes in [14] and [17]. Our scheme, as in [14], uses two separate keys for re-encryption.

\subsection{Our Results}

Let us briefly describe a C-PRE scheme. A C-PRE scheme involves a delegator (say user $\mathrm{U}_{\mathrm{i}}$ ), a delegatee (say user $U_{j}$ ) and a proxy. A message sent to $U_{i}$ with condition $w$ is encrypted by the sender using both $U_{i}$ 's public key and $w$. To re-encrypt the message to $U_{j}$, the proxy is given the re-encryption key $\left(\mathrm{rk}_{\mathrm{i} \rightarrow \mathrm{j}}\right)$ and the condition key $\left(\mathrm{ck}_{\mathrm{i}, \mathrm{w}}\right)$ corresponding to $\mathrm{w}$. Both the keys can be generated only by $\mathrm{U}_{\mathrm{i}}$. These two keys form the secret trapdoor to be used by the proxy to perform translation. Proxy will not be able to re-encrypt cipher texts for which the right condition key is not available. Thus $U_{i}$ can flexibly assign $U_{j}$ the decryption rights by setting condition keys properly. The scheme works in practice as follows: the message encrypted for $U_{i}$ is first handled by proxy and under appropriate conditions the proxy transforms the ciphertext into a ciphertext for $U_{j}$. However, proxy will obtain no information about the original message. While it is some what easier to design a PRE without pairing, designing C-PRE requires pairing based operations crucially. We have used a few constructions from [12] which drastically reduces the number of bilinear pairings. Table 1 compares the number of bilinear pairings and exponentiations between the scheme in [17] and our scheme.

Table 1. Computational Complexity Comparison

\begin{tabular}{|l|c|c|c|c|}
\hline \multirow{2}{*}{ Algorithm } & \multicolumn{2}{|c|}{ Scheme in [17] } & \multicolumn{2}{c|}{ Our Scheme } \\
\cline { 2 - 5 } & BP & EXP & BP & EXP \\
\hline Encryption case 1 & 1 & 4 & 0 & 0 \\
\hline Encryption case 2 & 1 & 3 & 1 & 6 \\
\hline Re-Encryption & 3 & 4 & 1 & 3 \\
\hline Decryption case 1 & 3 & 3 & 1 & 4 \\
\hline Decryption case 2 & 1 & 1 & 0 & 6 \\
\hline Total & 9 & 15 & 3 & 19 \\
\hline
\end{tabular}

BP - Bilinear Pairings, EXP - Exponentiations. 
International Journal of Network Security \& Its Applications (IJNSA), Vol.4, No.2, March 2012

Encryption case 1 refers to the encryption without the condition. Encryption case 2 refers to the encryption with the condition. Decryption case 1 refers to the decryption of the re-encrypted ciphertext (first level ciphertext) and Decryption case 2 refers to the decryption of the encrypted ciphertext (second level ciphertext).

Although the number of exponentiations in our scheme is slightly more, it is insignificant when compared to the reduction in number of bilinear pairings. Thus, our scheme is more efficient than the existing one. We then formally prove the security of our scheme. We have slightly modified the security model in [14], as discussed in Section 3.

The C-PRE scheme in [14] has a break as given in [17]. Scheme in [17] has combined the two keys into a single key. Having the keys separate has an advantage. The delegation power of the proxy can be controlled. One of the two keys can be given to the proxy for partial re-encryption and the other key can be given to a third party for full re-encryption. Since the scheme in [14] has a break, our scheme is the only existing scheme having this unique property.

\section{Preliminaries}

Bilinear Groups and Bilinear Pairings: Let $G$ and $G_{T}$ be two cyclic multiplicative groups with the same prime order $q$. A bilinear pairing is a map e : $G \times G \rightarrow G_{T}$ with the following properties.

- Bilinearity: We have $\hat{\mathrm{e}}\left(\mathrm{g}_{1}^{\mathrm{a}}, \mathrm{g}_{2}^{\mathrm{b}}\right)=\hat{\mathrm{e}}\left(\mathrm{g}_{1}, \mathrm{~g}_{2}\right)^{\mathrm{ab}} \forall \mathrm{g}_{1}, \mathrm{~g}_{2} \in \mathrm{G}$ and $\forall \mathrm{a}, \mathrm{b} \in Z_{\mathrm{q}}^{*}$;

- Non-degeneracy: There exist $g_{1}, g_{2} \in G$ such that $\hat{e}\left(g_{1}, g_{2}\right) \neq 1$;

- Computability: There exists an efficient algorithm to compute $\hat{\mathrm{e}}\left(\mathrm{g}_{1}, \mathrm{~g}_{2}\right) \forall \mathrm{g}_{1}, \mathrm{~g}_{2} \in \mathrm{G}$.

Modified Computational Diffie-Hellman Problem: Let $\mathrm{G}$ be a cyclic multiplicative group with a prime order $\mathrm{q}$. Let $\mathrm{g}$ be the generator of $\mathrm{G}$, The $\mathrm{mCDH}$ problem in $\mathrm{G}$ is as follows:

Given $\left(\mathrm{g}, \mathrm{g}^{\frac{1}{\mathrm{a}}}, \mathrm{g}^{\mathrm{a}}, \mathrm{g}^{\mathrm{b}}\right)$ for some $\mathrm{a}, \mathrm{b} \in \mathrm{Z}_{\mathrm{q}}^{*}$, compute $\mathrm{W}=\mathrm{g}^{\mathrm{ab}} \in \mathrm{G}$. An algorithm $\mathcal{A}$ has an advantage $\varepsilon$ in solving $\mathrm{mCDH}$ in $\mathrm{G}$ if

$$
\operatorname{Pr}\left[\mathcal{A}\left(\mathrm{g}, \mathrm{g}^{\frac{1}{\mathrm{a}}}, \mathrm{g}^{\mathrm{a}}, \mathrm{g}^{\mathrm{b}}\right)=\mathrm{g}^{\mathrm{ab}}\right] \geq \varepsilon
$$

where the probability is over the random choice of $a, b \in Z_{\mathrm{q}}^{*}$, the random choice of $g \in G$ and the random bits of $\mathcal{A}$.

Modified Computational Bilinear Diffie-Hellman Problem: Let $G$ and $G_{T}$ be two cyclic multiplicative groups with the same prime order q. Let e : $G \times G \rightarrow G_{T}$ be an admissible bilinear map and let $g$ be the generator of $G$. The $m C B D H$ problem in $\left(G, G_{T}, e\right)$ is as follows:

Given $\left(\mathrm{g}, \mathrm{g}^{\frac{1}{\mathrm{a}}}, \mathrm{g}^{\mathrm{a}}, \mathrm{g}^{\mathrm{b}}, \mathrm{g}^{\mathrm{c}}\right)$ for some $\mathrm{a}, \mathrm{b}, \mathrm{c} \in \mathrm{Z}_{\mathrm{q}}^{*}$, compute $\mathrm{W}=\hat{\mathrm{e}}(\mathrm{g}, \mathrm{g})^{\mathrm{abc}} \in \mathrm{G}_{\mathrm{T}}$. An algorithm $\mathcal{A}$ has an advantage $\in$ in solving $\mathrm{mCBDH}$ in $\left(\mathrm{G}, \mathrm{G}_{\mathrm{T}}, \mathrm{e}\right)$ if

$$
\operatorname{Pr}\left\lfloor\mathcal{A}\left(\mathrm{g}, \mathrm{g}^{\frac{1}{\mathrm{a}}}, \mathrm{g}^{\mathrm{a}}, \mathrm{g}^{\mathrm{b}}, \mathrm{g}^{\mathrm{c}}\right)=\hat{\mathrm{e}}(\mathrm{g}, \mathrm{g})^{\mathrm{abc}}\right\rfloor \geq \varepsilon
$$

where the probability is over the random choice of $a, b, c \in Z_{q}^{*}$, the random choice of $g \in G$ and the random bits of $\mathcal{A}$.

\section{Model of Conditional Proxy Re-Encryption}

We give the definitions and security notions for C-PRE systems in this section.

\subsection{Definition of C-PRE systems}

A unidirectional C-PRE scheme consists of seven algorithms which are described as follows: 
Global Setup $(\lambda)$ : The global setup algorithm takes a security parameter $\lambda$ as input and outputs the global parameters param. The parameters in param are implicitly given as input to the following algorithms.

KeyGen (i): The key generation algorithm takes the user index $i$ as input and generates a public $\mathrm{key}\left(\mathrm{pk}_{\mathrm{i}}\right)$ and a secret key $\left(\mathrm{sk}_{\mathrm{i}}\right)$ for user $\mathrm{U}_{\mathrm{i}}$.

ReKeyGen $\left(\mathrm{sk}_{\mathrm{i}}, \mathrm{pk}_{\mathrm{j}}\right)$ : The partial re-encryption key generation algorithm takes a secret key $\mathrm{sk}_{\mathrm{i}}$ and another public key $\mathrm{pk}_{\mathrm{j}}$ as input and outputs the partial re-encryption key $\mathrm{rk}_{\mathrm{i}} \rightarrow \mathrm{j}$. This algorithm is run by $U_{i}$. Here $s_{j}$ is not taken as input which indeed makes the scheme unidirectional.

CKeyGen $\left(\mathrm{sk}_{\mathrm{i}}, \mathrm{w}\right)$ : The condition key generation algorithm takes a secret key $\mathrm{sk}_{\mathrm{i}}$ and a condition $\mathrm{w}$ as input and outputs the condition key $\mathrm{ck}_{\mathrm{i}, \mathrm{w}}$. This algorithm is run by $\mathrm{U}_{\mathrm{i}}$.

Encrypt (pk, m, w): The encryption algorithm takes a public key pk, a message $\mathrm{m}$ and a condition $\mathrm{w}$ as input and outputs the ciphertext $\zeta$ associated with $\mathrm{w}$ under $\mathrm{pk}$. Here $\mathrm{m} \in \mathcal{M}$ where $\mathcal{M}$ denotes the message space.

ReEncrypt $\left(\mathrm{rk}_{\mathrm{i} \rightarrow \mathrm{j}}, \mathrm{ck}_{\mathrm{i}, \mathrm{w}}, \zeta_{\mathrm{i}}\right)$ : The re-encryption algorithm takes a partial re-encryption key $\mathrm{rk}_{\mathrm{i}} \rightarrow$ $\mathrm{j}$, a condition key $\mathrm{ck}_{\mathrm{i}, \mathrm{w}}$ associated with condition $\mathrm{w}$ and a ciphertext $\zeta_{\mathrm{i}}$ under the public key $\mathrm{pk}_{\mathrm{i}}$ as input and outputs the re-encrypted ciphertext $\zeta_{\mathrm{j}}$ under the public key $\mathrm{pk}_{\mathrm{j}}$. This algorithm is run by the proxy.

Decrypt (sk, $\zeta)$ : The decryption algorithm takes a secret key sk and a ciphertext $\zeta$ as input and outputs either a message $\mathrm{m} \in \mathcal{M}$ or the error symbol $\perp$.

Correctness: For any $\mathrm{m} \in \mathcal{M}$, any condition $\mathrm{w}$, any $\left(\mathrm{pk}_{\mathrm{i}}, \mathrm{sk}_{\mathrm{i}}\right) \leftarrow \operatorname{KeyGen}(\mathrm{i}),\left(\mathrm{pk}_{\mathrm{j}}, \mathrm{sk}_{\mathrm{j}}\right) \leftarrow$ KeyGen (j), and

$\zeta_{\mathrm{i}}=$ Encrypt $\left(\mathrm{pk}_{\mathrm{i}}, \mathrm{m}, \mathrm{w}\right)$,

$\operatorname{Pr}\left[\operatorname{Decrypt}\left(\mathrm{sk}_{\mathrm{i}}, \zeta_{\mathrm{i}}\right)=\mathrm{m}\right]=1$, and

$\operatorname{Pr}\left[\operatorname{Decrypt}\left(\mathrm{sk}_{\mathrm{j}}, \operatorname{ReEncrypt}\left(\mathrm{rk}_{\mathrm{i}, \mathrm{j}}, \mathrm{ck}_{\mathrm{i}, \mathrm{w}}, \zeta_{\mathrm{i}}\right)\right)=\mathrm{m}\right]=1$.

while for any other condition $w^{\prime}$ and user $j^{\prime}$ with $w^{\prime} \neq w$ and $j^{\prime} \neq j$, we have

$\operatorname{Pr}\left[\operatorname{Decrypt}\left(\mathrm{sk}_{\mathrm{j}}, \operatorname{ReEncrypt}\left(\mathrm{rk}_{\mathrm{i}, \mathrm{j}}, \mathrm{ck}_{\mathrm{i}, \mathrm{w}^{\prime}}, \zeta_{\mathrm{i}}\right)\right)=\perp\right]=1-\operatorname{neg}(\lambda)$

$\operatorname{Pr}\left[\operatorname{Decrypt}\left(\mathrm{sk}_{\mathrm{j}}, \operatorname{ReEncrypt}\left(\mathrm{rk}_{\mathrm{i}, \mathrm{j}^{\prime}}, \mathrm{ck}_{\mathrm{i}, \mathrm{w}}, \zeta_{\mathrm{i}}\right)\right)=\perp\right]=1-\operatorname{neg}(\lambda)$.

\subsection{Security Notions}

The following game between an adversary $\mathcal{A}$ and a challenger $C$ is used to define the semantic security of our C-PRE scheme against chosen ciphertext attacks.

Setup. $C$ takes a security parameter $\lambda$ and runs the algorithm GlobalSetup $(\lambda)$ and gives the resulting global parameters param to $\mathcal{A}$.

Phase 1. $\mathcal{A}$ adaptively issues queries $\mathrm{q}_{1}, \ldots, \mathrm{q}_{\mathrm{m}}$ where $\mathrm{q}_{\mathrm{i}}$ is one of the following:

- Uncorrupted key generation query: $C$ first runs algorithm KeyGen (i) to obtain the public/secret key pair $\left(\mathrm{pk}_{\mathrm{i}}, \mathrm{sk}_{\mathrm{i}}\right)$, and then gives $\mathrm{pk}_{\mathrm{i}}$ to $\mathcal{A}$.

- Corrupted key generation query: $C$ first runs algorithm KeyGen (j) to obtain the public/secret key pair $\left(\mathrm{pk}_{\mathrm{j}}, \mathrm{sk}_{\mathrm{j}}\right)$, and then gives $\left(\mathrm{pk}_{\mathrm{j}}, \mathrm{sk}_{\mathrm{j}}\right)$ to $\mathcal{A}$.

- $\quad$ Partial re-encryption key generation query $\left(\mathrm{pk}_{\mathrm{i}}, \mathrm{pk}_{\mathrm{j}}\right): C$ runs the algorithm ReKeyGen $\left(\mathrm{sk}_{\mathrm{i}}\right.$, $\mathrm{pk}_{\mathrm{j}}$ ) and returns the generated re-encryption key $\mathrm{rk}_{\mathrm{i} \rightarrow \mathrm{j}}$ to $\mathcal{A}$. Here $\mathrm{sk}_{\mathrm{i}}$ is the secret key corresponding to $\mathrm{pk}_{\mathrm{i}}$.

- Condition key generation query $\left(\mathrm{pk}_{\mathrm{i}}, \mathrm{w}\right)$ : $C$ runs the algorithm $C \operatorname{KeyGen}\left(\mathrm{sk}_{\mathrm{i}}, \mathrm{w}\right)$ and returns the generated condition key $\mathrm{ck}_{\mathrm{i}, \mathrm{w}}$ to $\mathcal{A}$. 
International Journal of Network Security \& Its Applications (IJNSA), Vol.4, No.2, March 2012

- Re-encryption query $\left(\mathrm{pk}_{\mathrm{i}}, \mathrm{pk}_{\mathrm{j}}, \mathrm{w}, \zeta_{\mathrm{i}}\right): C$ runs the algorithm $\operatorname{ReEncrypt}\left(\operatorname{ReKeyGen}\left(\mathrm{sk}_{\mathrm{i}}, \mathrm{pk}_{\mathrm{j}}\right)\right.$, $\left.\mathrm{CKeyGen}\left(\mathrm{sk}_{\mathrm{i}}, \mathrm{w}\right), \zeta_{\mathrm{i}}\right)$ and returns the generated ciphertext $\zeta_{\mathrm{j}}$ to A.

- Decryption query $(\mathrm{pk}, \mathrm{w}, \zeta)$ or $\left(\mathrm{p}_{\mathrm{k}}, \zeta\right): C$ runs the algorithm Decrypt(sk, $\left.\zeta\right)$ and returns its result to $\mathcal{A}$. Here (pk, w, $\zeta$ ) and $(\mathrm{pk}, \zeta$ ) are queries on original ciphertexts and re-encrypted ciphertexts respectively.

For the last four queries it is required that $\mathrm{pk}, \mathrm{pk}_{\mathrm{i}}$ and $\mathrm{pk}_{\mathrm{j}}$ are generated beforehand by the KeyGen algorithm.

Challenge. Once $\mathcal{A}$ decides Phase 1 is over, it outputs a target public key $\mathrm{pk}_{\mathrm{i}^{*}}$, a target condition $\mathrm{w}^{*}$ and two equal-length plaintexts $\mathrm{m}_{0}, \mathrm{~m}_{1} \in \mathcal{M}$. $C$ flips a random coin $\delta \in\{0,1\}$, and sets the challenge ciphertext to be $\zeta^{*}=\operatorname{Encrypt}\left(\mathrm{pk}_{\mathrm{i}^{*}}, \mathrm{~m}_{\delta}, \mathrm{w}^{*}\right)$, which is sent to $\mathcal{A}$.

Phase 2: $\mathcal{A}$ adaptively issues queries as in Phase 1, and $C$ answers them as before.

Guess: Finally, $\mathcal{A}$ outputs a guess $\delta^{\prime} \in\{0,1\}$ and wins the game if $\delta^{\prime}=\delta$. Adversary $\mathcal{A}$ is subject to the following restrictions during the above game.

1. $\mathcal{A}$ cannot issue corrupted key generation queries on $\mathrm{i}^{*}$ to obtain the target secret key $\mathrm{sk}_{\mathrm{i}}$.

2. $\mathcal{A}$ can issue decryption queries on neither $\left(\mathrm{pk}_{\mathrm{i}^{*}}, \mathrm{w}^{*}, \zeta^{*}\right)$ nor $\left(\mathrm{pk}_{\mathrm{j}}, \operatorname{ReEncrypt}\left(\mathrm{rk}_{\mathrm{i}^{*}} \rightarrow \mathrm{j}\right.\right.$,

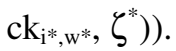

3. $\mathcal{A}$ cannot issue re-encryption queries on $\left(\mathrm{pk}_{\mathrm{i}^{*}}, \mathrm{pk}_{\mathrm{j}}, \mathrm{w}^{*}, \zeta^{*}\right)$ if $\mathrm{pk}_{\mathrm{j}}$ appears in a previous corrupted key generation query.

4. $\mathcal{A}$ cannot obtain the partial re-encryption key $\mathrm{rk}_{\mathrm{i}^{*} \rightarrow \mathrm{j}}$ if $\mathrm{pk}_{\mathrm{j}}$ appears in a previous corrupted key generation query.

We refer to the above adversary $\mathcal{A}$ as an IND-CPRE-CCA adversary. $\mathcal{A}$ 's advantage in attacking our CPRE scheme is defined as $\operatorname{Adv}_{\mathrm{C}-\mathrm{PRE}, \mathcal{A}}^{\mathrm{IND}-\mathrm{CPE}-\mathrm{CCA}}=\left|\operatorname{Pr}\left[\delta^{\prime}=\delta\right]-1 / 2\right|$, where the probability is taken over the random coins consumed by the adversary and the challenger. As in [14], we also distinguish between two types of IND-CPRE-CCA adversaries as follows:

- Type I IND-CPRE-CCA adversary: In the game, adversary $\mathcal{A}$ does not obtain the reencryption key $\mathrm{rk}_{\mathrm{i}^{*} \rightarrow \mathrm{j}}$ with $\mathrm{pk}_{\mathrm{j}}$ corrupted.

- Type II IND-CPRE-CCA adversary: In the game, adversary $\mathcal{A}$ does not obtain both the condition key $\mathrm{ck}_{\mathrm{i}^{*}, \mathrm{w}^{*}}$ and the re-encryption key $\mathrm{rk}_{\mathrm{i}^{*} \rightarrow \mathrm{j}}$ with $\mathrm{pk}_{\mathrm{j}}$ corrupted.

\section{AN EFFICIENT C-PRE SCHEME}

Here we present our efficient C-PRE scheme and then prove its security.

\subsection{Construction}

Our proposed scheme consists of the following seven main algorithms and one auxiliary algorithm for checking the validity of the ciphertext.

Global Setup $(\lambda)$ : This algorithm takes the security parameter $\lambda$ as input. Then two primes $p$ and $\mathrm{q}$ are chosen such that $\mathrm{q} \mid \mathrm{p}-1$ where $\mathrm{q}$ is a $\lambda$ bit prime. Then the algorithm generates ( $\mathrm{q}, \mathrm{G}$, $G_{T}, e$ ) where $G$ and $G_{T}$ are two cyclic groups with prime order $q$ and e is a bilinear pairing e : $G$ $\times G \rightarrow G_{T}$. Let $g$ be the generator of group $G$, which is a subgroup of $Z_{q}^{*}$ with order $q$. Choose hash functions as follows: 
International Journal of Network Security \& Its Applications (IJNSA), Vol.4, No.2, March 2012

$\mathrm{H}_{1}:\{0,1\}^{l_{0}} \times\{0,1\}^{l_{1}} \rightarrow \mathrm{Z}_{q}^{*}, \mathrm{H}_{2}:\{0,1\}^{*} \rightarrow \mathrm{Z}_{q}^{*}, \mathrm{H}_{3}: \mathrm{G} \rightarrow\{0,1\}^{l_{0}+l_{1}}, \mathrm{H}_{4}:\{0,1\}^{*} \rightarrow \mathrm{Z}_{q}^{*}$, $\mathrm{H}_{5}: \mathrm{G} \rightarrow \mathrm{Z}_{q}^{*}, \mathrm{H}_{6}:\{0,1\}^{*} \times \mathrm{G} \times \mathrm{G} \rightarrow \mathrm{G}$, and $\mathrm{H}_{7}: \mathrm{G}_{\mathrm{T}} \rightarrow\{0,1\}^{l_{0}+l_{1}}$.

param $=\left(\left(\mathrm{q}, \mathrm{G}, \mathrm{G}_{\mathrm{T}}, \mathrm{e}\right), \mathrm{g}, \mathrm{H}_{1}, \ldots, \mathrm{H}_{7}\right) . l_{0}$ and $l_{1}$ are determined by $\lambda$ and the message space $\mathcal{M}$ is $\{0,1\}^{l_{0}}$.

KeyGen (i): This algorithm randomly picks $\mathrm{sk}_{\mathrm{i}}=\left(\mathrm{x}_{\mathrm{i}, 1}, \mathrm{x}_{\mathrm{i}, 2} \stackrel{\$}{\leftarrow} \mathrm{Z}_{q}^{*}\right)$ and sets $\mathrm{pk}_{\mathrm{i}}=\left(\mathrm{g}^{\mathrm{x}_{\mathrm{i}, 1}}, \mathrm{~g}^{\mathrm{x}_{\mathrm{i} 2}}\right)$.

$\operatorname{ReKeyGen}\left(\mathrm{sk}_{\mathrm{i}}, \mathrm{pk}_{\mathrm{j}}\right)$ : The re-encryption key $\mathrm{rk}_{\mathrm{i} \rightarrow \mathrm{j}}$ is generated as follows:

1. Pick $\mathrm{h} \stackrel{\$}{\leftarrow}\{0,1\}^{l_{0}}$ and $\pi \stackrel{\$}{\leftarrow}\{0,1\}^{l_{1}}$ and compute $\mathrm{v}=\mathrm{H}_{1}(\mathrm{~h}, \pi)$.

2. Compute $\mathrm{V}=\mathrm{g}^{\mathrm{v}}$ and $\mathrm{W}=\mathrm{H}_{3}\left(\mathrm{pk}_{\mathrm{j}, 2}^{\mathrm{v}}\right) \oplus(\mathrm{h} \| \pi)$.

3. Compute $\mathrm{rk}_{\mathrm{i} \rightarrow \mathrm{j}}^{(1)}=\frac{\mathrm{h}}{\mathrm{x}_{\mathrm{i}, 1} \mathrm{H}_{5}\left(\mathrm{pk}_{\mathrm{i}, 2}\right)+\mathrm{x}_{\mathrm{i}, 2}}$ and return $\mathrm{rk}_{\mathrm{i} \rightarrow \mathrm{j}}=\left(\mathrm{rk}_{\mathrm{i} \rightarrow \mathrm{j}}^{(1)}, \mathrm{V}, \mathrm{W}\right)$.

CKeyGen( $\left.\mathrm{sk}_{\mathrm{i}}, \mathrm{w}\right)$ : This algorithm outputs the condition key $\mathrm{ck}_{\mathrm{i}, \mathrm{w}}=\mathrm{H}_{6}\left(\mathrm{w}, \mathrm{pk}_{\mathrm{i}}\right)^{\frac{1}{\mathrm{x}_{\mathrm{i}, 1}}}$.

$\operatorname{Encrypt}\left(\mathrm{pk}_{\mathrm{i}}, \mathrm{m}, \mathrm{w}\right)$ : This algorithm encrypts a message $\mathrm{m}$ with condition $\mathrm{w}$ for $\mathrm{pk}_{\mathrm{i}}$ as follows:

1. Pick $\mathrm{s}, \mathrm{z} \stackrel{\$}{\leftarrow} \mathrm{Z}_{\mathrm{q}}^{*}$ and compute $\mathrm{B}=\mathrm{pk}_{\mathrm{i}, 1}^{\mathrm{s}}$ and $\mathrm{D}=\mathrm{pk}_{\mathrm{i}, 1}^{\mathrm{z}}$.

2. Pick $\mathrm{r}^{\prime} \stackrel{\$}{\leftarrow}\{0,1\}^{l_{1}}$. Compute $\mathrm{r}=\mathrm{H}_{2}\left(\mathrm{~m}, \mathrm{r}^{\prime}, \mathrm{pk}_{\mathrm{i}}, \mathrm{w}\right)$ and $\mathrm{A}=\left(\mathrm{pk}_{\mathrm{i}, 1}^{\mathrm{H}_{5}\left(\mathrm{pk}_{\mathrm{i}, 2}\right)} \mathrm{pk}_{\mathrm{i}, 2}\right)^{\mathrm{r}}$.

3. Compute $\mathrm{C}=\mathrm{H}_{3}\left(\mathrm{~g}^{\mathrm{r}}\right) \oplus\left(\mathrm{m} \| \mathrm{r}^{\prime}\right) \oplus \mathrm{H}_{7}\left(\hat{\mathrm{e}}\left(\mathrm{g}, \mathrm{H}_{6}\left(\mathrm{w}, \mathrm{pk}_{\mathrm{i}}\right)\right)^{\mathrm{s}}\right)$.

4. Compute $\mathrm{E}=\mathrm{s}+\mathrm{zH}_{4}(\mathrm{~A}, \mathrm{~B}, \mathrm{C}, \mathrm{D}) \bmod \mathrm{q}$.

5. Output the ciphertext $\zeta_{\mathrm{i}}=(\mathrm{A}, \mathrm{B}, \mathrm{C}, \mathrm{D}, \mathrm{E})$.

Validity(): This algorithm implicitly takes all the inputs of the calling algorithm as its input and works as follows:

If $\mathrm{pk}_{\mathrm{i}, 1}^{\mathrm{E}} \neq \mathrm{B} \cdot \mathrm{D}^{\mathrm{H}_{4}(\mathrm{~A}, \mathrm{~B}, \mathrm{C}, \mathrm{D})}$ return $\perp$.

$\operatorname{ReEncrypt}\left(\mathrm{rk}_{\mathrm{i} \rightarrow \mathrm{j}}, \mathrm{ck}_{\mathrm{i}, \mathrm{w}}, \zeta_{\mathrm{i}}, \mathrm{pk}_{\mathrm{i}}, \mathrm{pk}_{\mathrm{j}}\right)$ : This algorithm re-encrypts $\zeta_{\mathrm{i}}$ to $\zeta_{\mathrm{j}}$ as follows:

1. Return $\perp$ if Validity() returns $\perp$.

2. Compute $\mathrm{A}^{\prime}=\mathrm{A}^{\mathrm{rk} \mathrm{k}_{\mathrm{i} \rightarrow \mathrm{j}}^{(1)}}$ and $\mathrm{C}^{\prime}=\mathrm{C} \oplus \mathrm{H}_{7}\left(\hat{\mathrm{e}}\left(\mathrm{B}, \mathrm{ck}_{\mathrm{i}, \mathrm{w}}\right)\right)$.

3. Output the transformed ciphertext as $\zeta_{\mathrm{j}}=\left(\mathrm{A}^{\prime}, \mathrm{C}^{\prime}, \mathrm{V}, \mathrm{W}\right)$.

$\operatorname{Decrypt}\left(\mathrm{sk}_{\mathrm{i}}, \zeta_{\mathrm{i}}\right)$ : Parse the ciphertext $\zeta_{\mathrm{i}}$. Decryption of $\zeta_{\mathrm{i}}$ is done as follows:

- $\zeta$ is the original ciphertext in the form $\zeta=(A, B, C, D, E)$.

1. Return $\perp$ if Validity() returns $\perp$.

2. Compute $\left(m \| r^{\prime}\right)=C \oplus H_{3}\left(\mathrm{~A}^{\frac{1}{\mathrm{x}_{1,1} \mathrm{H}_{5}\left(\frac{1}{\left(\mathrm{k}_{\mathrm{i}, 2}\right)+\mathrm{x}_{\mathrm{i}, 2}}\right)}}\right) \oplus \mathrm{H}_{7}\left(\hat{\mathrm{e}}\left(\mathrm{B}, \mathrm{H}_{6}\left(\mathrm{w}, \mathrm{pk}_{\mathrm{i}}\right)\right)^{\frac{1}{\mathrm{x}_{\mathrm{i}, 1}}}\right)$.

3. If $\mathrm{A}=\left(\mathrm{pk}_{\mathrm{i}, 1}^{\mathrm{H}_{5}\left(\mathrm{pk}_{\mathrm{i}, 2}\right)} \mathrm{pk}_{\mathrm{i}, 2}\right)^{\mathrm{H}_{2}\left(\mathrm{~m}, \mathrm{r}^{\prime}, \mathrm{pk}_{\mathrm{i}}, \mathrm{w}\right)}$ holds, return $\mathrm{m}$; else return $\perp$.

- $\zeta$ is the re-encrypted ciphertext in the form $\zeta=\left(\mathrm{A}^{\prime}, \mathrm{C}^{\prime}, \mathrm{V}, \mathrm{W}\right)$.

1. Compute $(\mathrm{h} \| \pi)=\mathrm{W} \oplus \mathrm{H}_{3}\left(\mathrm{~V}^{\mathrm{sk}} \mathrm{i}_{\mathrm{i}, 2}\right)$ and $\left(\mathrm{m} \| \mathrm{r}^{\prime}\right)=\mathrm{C}^{\prime} \oplus \mathrm{H}_{3}\left(\mathrm{~A}^{\prime \frac{1}{h}}\right)$.

2. If $\mathrm{V}=\mathrm{g}^{\mathrm{H}_{1}(\mathrm{~h}, \pi)}$ and $\mathrm{A}^{\prime}=\mathrm{g}^{\mathrm{hH}_{2}\left(\mathrm{~m}, \mathrm{r}^{\prime}, \mathrm{pk}_{\mathrm{i}}, \mathrm{w}\right)}$ hold, return m; else return $\perp$. 
International Journal of Network Security \& Its Applications (IJNSA), Vol.4, No.2, March 2012

Correctness: The proxy must have both the right re-encryption key and the condition key to reencrypt a ciphertext to the delegatee. Otherwise, the delegatee will not be able to decrypt the ciphertext with non-negligible probability. Suppose a proxy has the re-encryption key $\mathrm{rk}_{\mathrm{i} \rightarrow \mathrm{j}}$ and the condition key $\mathrm{ck}_{\mathrm{i}, \mathrm{w}^{\prime}}\left(\mathrm{w}^{\prime} \neq \mathrm{w}\right)$, he will generate the re-encrypted ciphertext $\zeta_{\mathrm{j}}=\left(\mathrm{A}^{\prime}, \mathrm{C}^{\prime}, \mathrm{V}, \mathrm{W}\right)$ as

$$
\begin{aligned}
\mathrm{A}^{\prime} & =\mathrm{g}^{\mathrm{rh}} \\
\mathrm{C}^{\prime} & =\mathrm{H}_{3}\left(\mathrm{~g}^{\mathrm{r}}\right) \oplus\left(\mathrm{m} \| \mathrm{r}^{\prime}\right) \oplus \mathrm{H}_{7}\left(\hat{\mathrm{e}}\left(\mathrm{g}, \mathrm{H}_{6}\left(\mathrm{w}, \mathrm{pk}_{\mathrm{i}}\right)\right)^{\mathrm{s}}\right) \oplus \mathrm{H}_{7}\left(\hat{\mathrm{e}}\left(\mathrm{B}, \mathrm{ck}_{\mathrm{i}, \mathrm{w}^{\prime}}\right)\right) \\
& =\mathrm{H}_{3}\left(\mathrm{~g}^{\mathrm{r}}\right) \oplus\left(\mathrm{m} \| \mathrm{r}^{\prime}\right) \oplus \mathrm{H}_{7}\left(\hat{\mathrm{e}}\left(\mathrm{g}, \mathrm{H}_{6}\left(\mathrm{w}, \mathrm{pk}_{\mathrm{i}}\right)\right)^{\mathrm{s}}\right) \oplus \mathrm{H}_{7}\left(\hat{\mathrm{e}}\left(\mathrm{g}^{\mathrm{sx}, 1,1}, \mathrm{H}_{6}\left(\mathrm{w}^{\prime}, \mathrm{pk}_{\mathrm{i}}\right)^{\frac{1}{\mathrm{x}, 1}}\right)\right) \\
& =\mathrm{H}_{3}\left(\mathrm{~g}^{\mathrm{r}}\right) \oplus\left(\mathrm{m} \| \mathrm{r}^{\prime}\right) \oplus \mathrm{H}_{7}\left(\hat{\mathrm{e}}\left(\mathrm{g}, \mathrm{H}_{6}\left(\mathrm{w}, \mathrm{pk}_{\mathrm{i}}\right)\right)^{\mathrm{s}}\right) \oplus \mathrm{H}_{7}\left(\hat{\mathrm{e}}\left(\mathrm{g}, \mathrm{H}_{6}\left(\mathrm{w}^{\prime}, \mathrm{pk}_{\mathrm{i}}\right)\right)^{\mathrm{s}}\right) \\
\mathrm{V} & =\mathrm{g}^{\mathrm{v}} \\
\mathrm{W} & =\mathrm{H}_{3}\left(\mathrm{pk}_{\mathrm{j}, 2}^{\mathrm{v}}\right) \oplus(\mathrm{h} \| \pi) .
\end{aligned}
$$

Note that the two $\mathrm{H}_{7}$ terms do not cancel each other implying that $\mathrm{C}^{\prime} \oplus \mathrm{H}_{3}\left(\mathrm{~A}^{\frac{1}{\mathrm{~g}}}\right)$ in the decryption algorithm will not reveal the message $\mathrm{m}$ with overwhelming probability. The resulting value will also not pass the condition checks. Hence the delegatee cannot decrypt the re-encrypted ciphertext with high probability.

Security intuitions: It is impossible for the adversary to manipulate the ciphertext. This is because the validity of the original ciphertext can be publicly verified by the Validity() algorithm. Thus our scheme can ensure chosen-ciphertext security. Even if the conditional key $\mathrm{w}$ is changed to another value $\mathrm{w}^{\prime}$ by the adversary, the scheme is secure because $\mathrm{w}$ is a parameter for $\mathrm{H}_{2}$ and when $w$ changes the value of $\mathrm{r}$ also changes.

\subsection{Security}

The proposed C-PRE scheme is IND-CPRE-CCA secure in random oracle model. This follows directly from Theorem 1 and Theorem 2 .

Theorem 1. Our scheme is IND-CPRE-CCA secure in the random oracle model, assuming the $\mathrm{mCDH}$ assumption holds in group $\mathrm{G}$ and the Schnorr signature is EUF-CMA secure. Concretely, if there exists a Type I adversary $\mathcal{A}$, who asks at most $\mathrm{q}_{\mathrm{H}_{\mathrm{i}}}$ random oracle queries to $\mathrm{H}_{\mathrm{i}}$ with $\mathrm{i} \in\{1,2, \ldots, 7\}$, and breaks the $\left(\mathrm{t}, \mathrm{q}_{\mathrm{u}}, \mathrm{q}_{\mathrm{c}}, \mathrm{q}_{\mathrm{rk}}, \mathrm{q}_{\mathrm{ck}}, \mathrm{q}_{\mathrm{re}}, \mathrm{q}_{\mathrm{d}}, \varepsilon\right)$-IND-CPRE-CCA of our scheme, then, for any $0<\psi<\varepsilon$, there exists

1. either an algorithm $B$ which can break the $\left(\mathrm{t}^{\prime}, \varepsilon^{\prime}\right)$-mCDH assumption in $\mathrm{G}$ with

$$
\begin{aligned}
\mathrm{t}^{\prime} \leq \mathrm{t}+\left(\mathrm{q}_{\mathrm{H}_{1}}+\mathrm{q}_{\mathrm{H}_{2}}+\mathrm{q}_{\mathrm{H}_{3}}+\mathrm{q}_{\mathrm{H}_{4}}+\mathrm{q}_{\mathrm{H}_{5}}+\mathrm{q}_{\mathrm{H}_{6}}+\mathrm{q}_{\mathrm{H}_{7}}+\mathrm{q}_{\mathrm{u}}+\mathrm{q}_{\mathrm{c}}+\mathrm{q}_{\mathrm{rk}}+\mathrm{q}_{\mathrm{ck}}+\mathrm{q}_{\mathrm{re}}+\mathrm{q}_{\mathrm{d}}\right) O(1) \\
\quad+\left(2 \mathrm{q}_{\mathrm{c}}+2 \mathrm{q}_{\mathrm{u}}+6 \mathrm{q}_{\mathrm{rk}}+\mathrm{q}_{\mathrm{ck}}+\left(\mathrm{q}_{\mathrm{re}}+1\right)\left(2 \mathrm{q}_{\mathrm{d}}+\left(2 \mathrm{q}_{\mathrm{H}_{2}}+2 \mathrm{q}_{\mathrm{H}_{3}}\right) \mathrm{q}_{\mathrm{d}}\right)\right) \mathrm{t}_{\mathrm{exp}}+\left(\mathrm{q}_{\mathrm{re}}+\mathrm{q}_{\mathrm{d}}\right) \mathrm{t}_{\mathrm{p}} \\
\varepsilon^{\prime} \geq \frac{1}{\mathrm{q}_{\mathrm{H}_{3}}}\left(\frac{\varepsilon-\psi}{\varepsilon\left(1+\mathrm{q}_{\mathrm{rk}}\right)}-\frac{\mathrm{q}_{\mathrm{H}_{2}}+\mathrm{q}_{\mathrm{H}_{4}}+\left(\mathrm{q}_{\mathrm{H}_{2}}+\mathrm{q}_{\mathrm{H}_{3}}\right)\left(\mathrm{q}_{\mathrm{re}}+\mathrm{q}_{\mathrm{d}}\right)}{2^{\mathrm{l}_{0}+\mathrm{l}_{1}}}-\frac{2\left(\mathrm{q}_{\mathrm{re}}+\mathrm{q}_{\mathrm{d}}\right)}{\mathrm{q}}\right)
\end{aligned}
$$

where $t_{\text {exp }}$ denotes the running time of an exponentiation in group $G$ and $t_{p}$ denotes the running time of a pairing in groups $\left(\mathrm{G}, \mathrm{G}_{\mathrm{T}}\right)$.

2. or an attacker who breaks the EUF-CMA security of the Schnorr signature with advantage $\psi$ within time $t^{\prime}$. 
International Journal of Network Security \& Its Applications (IJNSA), Vol.4, No.2, March 2012

PROOF. Without loss of generality, we assume that the Schnorr signature is $\left(\mathrm{t}^{\prime}, \psi\right)$-EUF-CMA secure for some probability $0<\psi<\varepsilon$. If there exists a t-time adversary $\mathcal{A}$ who can break the IND-CPRE-CCA security of our scheme with advantage $\varepsilon-\psi$, then we show how to construct an algorithm $\mathscr{B}$ which can break the $\left(\mathrm{t}^{\prime}, \varepsilon^{\prime}\right)-\mathrm{mCDH}$ assumption in $\mathrm{G}$. Suppose $\mathcal{B}$ is given as input a challenge tuple $\left(\mathrm{g}, \mathrm{g}^{\frac{1}{\mathrm{a}}}, \mathrm{g}^{\mathrm{a}}, \mathrm{g}^{\mathrm{b}}\right)$ with unknown $\mathrm{a}, \mathrm{b} \stackrel{\$}{\leftarrow} Z_{\mathrm{q}}^{*}$. Algorithm $\mathfrak{B}^{\prime}$ s goal is to output $\mathrm{g}^{\mathrm{ab}}$. Algorithm $\mathscr{B}$ first gives $(\mathrm{q}, \mathrm{G}, \mathrm{g}$, $\left.\mathrm{H}_{1}, \ldots, \mathrm{H}_{7}, l_{0}, l_{1}\right)$ to $\mathcal{A}$. Next, $\mathscr{B}$ acts as a challenger and plays the IND-CPRE-CCA game with adversary $\mathcal{A}$ in the following way:

Hash Oracle Queries: At any time adversary $\mathcal{A}$ can issue random oracle queries $\mathrm{H}_{\mathrm{i}}$ with $\mathrm{i} \in\{1$, $\ldots, 7\}$. Algorithm $\mathscr{B}$ maintains seven hash lists $\mathrm{H}_{\mathrm{i}}^{\text {list }}$ with $\mathrm{i} \in\{1, \ldots, 7\}$ which are initially empty, and responds as below:

1. $\mathrm{H}_{1}$ queries: If $\mathrm{H}_{1}(\mathrm{~h}, \pi)$ has appeared on the $\mathrm{H}_{\mathrm{i}}^{\text {list }}$ in a tuple $(\mathrm{h}, \pi, \mathrm{v})$, return the predefined value v. Otherwise, choose $\mathrm{v} \leftarrow \mathrm{Z}_{\mathrm{q}}^{*}$ and add tuple $(\mathrm{h}, \pi, \mathrm{v})$ to $\mathrm{H}_{\mathrm{i}}^{\text {list }}$ and respond with $\mathrm{H}_{1}(\mathrm{~h}$, $\pi)=\mathrm{v}$.

2. $\mathrm{H}_{2}$ queries: If $\mathrm{H}_{2}\left(\mathrm{~m}, \mathrm{r}^{\prime}, \mathrm{pk}_{\mathrm{i}}, \mathrm{w}\right)$ has appeared on the $\mathrm{H}_{2}^{\text {list }}$ in a tuple $\left(\mathrm{m}, \mathrm{r}^{\prime}, \mathrm{pk}, \mathrm{w}, \mathrm{r}\right)$, return the predefined value $r$. Otherwise, choose $\mathrm{r} \stackrel{\$}{\leftarrow} \mathrm{Z}_{\mathrm{q}}^{*}$ and add tuple $\left(\mathrm{m}, \mathrm{r}^{\prime}, \mathrm{pk}_{\mathrm{i}}, \mathrm{w}, \mathrm{r}\right)$ to $\mathrm{H}_{2}^{\text {list }}$ and respond with $\mathrm{H}_{2}\left(\mathrm{~m}, \mathrm{r}^{\prime}, \mathrm{pk}_{\mathrm{i}}, \mathrm{w}\right)=\mathrm{r}$.

3. $\mathrm{H}_{3}$ queries: If $\mathrm{H}_{3}(\mathrm{R})$ has appeared on the $\mathrm{H}_{3}^{\text {list }}[\mathrm{R} \in \mathrm{G}]$ in a tuple $(\mathrm{R}, \beta)$, return the predefined value $\beta$. Otherwise, choose $\beta \stackrel{\$}{\leftarrow}\{0,1\}^{l_{0}+l_{1}}$, add tuple $(\mathrm{R}, \beta)$ to $\mathrm{H}_{3}^{\text {list }}$ and respond with $\mathrm{H}_{3}(\mathrm{R})=\beta$.

4. $\mathrm{H}_{4}$ queries: If $\mathrm{H}_{4}(\mathrm{~A}, \mathrm{~B}, \mathrm{C}, \mathrm{D})$ has appeared on the $\mathrm{H}_{4}^{\text {list }}$ in a tuple $(\mathrm{A}, \mathrm{B}, \mathrm{C}, \mathrm{D}, \gamma)$, return the predefined value $\gamma$. Otherwise, choose $\gamma \stackrel{\$}{\leftarrow} Z_{\mathrm{q}}^{*}$, add tuple $(\mathrm{A}, \mathrm{B}, \mathrm{C}, \mathrm{D}, \gamma)$ to $\mathrm{H}_{4}^{\text {list }}$ and respond with $\mathrm{H}_{4}(\mathrm{~A}, \mathrm{~B}, \mathrm{C}, \mathrm{D})=\gamma$.

5. $\mathrm{H}_{5}$ queries: If $\mathrm{H}_{5}(\mathrm{pk})$ has appeared on the $\mathrm{H}_{5}^{\text {list }}$ in a tuple $(\mathrm{pk}, \tau)$, return the predefined value $\tau$. Otherwise, choose $\tau \stackrel{\$}{\leftarrow} Z_{\mathrm{q}}^{*}$, add tuple $(\mathrm{pk}, \tau)$ to $\mathrm{H}_{5}^{\text {list }}$ and respond with $\mathrm{H}_{5}(\mathrm{pk})=$ $\tau$.

6. $\mathrm{H}_{6}$ queries: If $\mathrm{H}_{6}(\mathrm{w}, \mathrm{pk})$ has appeared on the $\mathrm{H}_{6}^{\text {list }}$ in a tuple $(\mathrm{w}, \mathrm{pk}, \mathrm{t}, \mathrm{S})$, return the predefined value $S$. Otherwise, choose $\mathrm{t} \stackrel{\$}{\leftarrow} Z_{\mathrm{q}}^{*}$, compute $\mathrm{S}=\mathrm{g}^{\mathrm{t}}$, add the tuple $(\mathrm{w}, \mathrm{pk}, \mathrm{t}, \mathrm{S})$ to $\mathrm{H}_{6}^{\text {list }}$ and respond with $\mathrm{H}_{6}(\mathrm{w}, \mathrm{pk})=\mathrm{S}$.

7. $\mathrm{H}_{7}$ queries: If $\mathrm{H}_{7}(\mathrm{U})$ has appeared on the $\mathrm{H}_{7}^{\text {list }}\left[\mathrm{U} \in \mathrm{G}_{\mathrm{T}}\right]$ in a tuple $(\mathrm{U}, \eta)$, return the predefined value $\eta$. Otherwise, choose $\eta \stackrel{\$}{\leftarrow}\{0,1\}^{l_{0}+l_{1}}$, add tuple (U, $\left.\eta\right)$ to $\mathrm{H}_{7}^{\text {list }}$ and respond with $\mathrm{H}_{7}(\mathrm{U})=\eta$.

Phase 1. In this phase, adversary $\mathcal{A}$ issues a series of queries subject to the restrictions of the Type I IND-CPRE-CCA game. $\mathcal{B}$ maintains three lists $\mathrm{K}^{\text {list }}, \mathrm{R}^{\text {list }}$ and $\mathrm{C}^{\text {list }}$ which are initially empty, and answers these queries for $\mathcal{A}$ as follows: 
International Journal of Network Security \& Its Applications (IJNSA), Vol.4, No.2, March 2012

- Uncorrupted key generation query. $B$ picks $\mathrm{x}_{\mathrm{i}, 1}, \mathrm{x}_{\mathrm{i}, 2} \stackrel{\$}{\leftarrow} \mathrm{Z}_{\mathrm{q}}^{*}$. Next, using the Coron's technique [6], it flips a biased coin $c_{i} \in\{0,1\}$ that yields 1 with probability $\theta$ and 0 otherwise. If $c_{i}=1$, it defines $\mathrm{pk}_{\mathrm{i}}=\left(\mathrm{g}^{\mathrm{x}_{\mathrm{i}, 1}}, \mathrm{~g}^{\mathrm{x}_{\mathrm{i}, 2}}\right)$; else $\mathrm{pk}_{\mathrm{i}}=\left(\left(\mathrm{g}^{\frac{1}{a}}\right)^{\mathrm{x}_{\mathrm{i}, 1}},\left(\mathrm{~g}^{\frac{1}{a}}\right)^{\mathrm{x}_{\mathrm{i}, 2}}\right)$. Then, it adds the tuple $\left(\mathrm{pk}_{\mathrm{i}}, \mathrm{x}_{\mathrm{i}, 1}, \mathrm{x}_{\mathrm{i}, 2}, \mathrm{c}_{\mathrm{i}}\right)$ to $\mathrm{K}^{\text {list }}$ and returns $\mathrm{pk}_{\mathrm{i}}$.

- Corrupted key generation query. $\mathcal{B}$ picks $\mathrm{x}_{\mathrm{i}, 1}, \mathrm{x}_{\mathrm{i}, 2} \stackrel{\$}{\leftarrow} \mathrm{Z}_{\mathrm{q}}^{*}$ and defines $\mathrm{pk}_{\mathrm{i}}=\left(\mathrm{g}^{\mathrm{x}_{\mathrm{i}, 1}}, \mathrm{~g}^{\mathrm{x}_{\mathrm{i}, 2}}\right), \mathrm{c}_{\mathrm{i}}$ $=$ ' - '. Then, it adds the tuple $\left(\mathrm{pk}_{\mathrm{i}}, \mathrm{x}_{\mathrm{i}, 1}, \mathrm{x}_{\mathrm{i}, 2}, \mathrm{c}_{\mathrm{i}}\right)$ to $\mathrm{K}^{\text {list }}$ and returns $\left(\mathrm{pk}_{\mathrm{i}},\left(\mathrm{x}_{\mathrm{i}, 1}, \mathrm{x}_{\mathrm{i}, 2}\right)\right)$.

- Re-encryption key generation query $\left(\mathrm{pk}_{\mathrm{i}}, \mathrm{pk}_{\mathrm{j}}\right)$. If $\mathrm{R}^{\text {list }}$ has an entry for $\left(\mathrm{pk}_{\mathrm{i}}, \mathrm{pk}_{\mathrm{j}}\right)$, return the predefined re-encryption key to $\mathcal{A}$. Otherwise, algorithm $\mathscr{B}$ acts as follows:

1. Recover tuples $\left(\mathrm{pk}_{\mathrm{i}}, \mathrm{x}_{\mathrm{i}, 1}, \mathrm{x}_{\mathrm{i}, 2}, \mathrm{c}_{\mathrm{i}}\right)$ and $\left(\mathrm{pk}_{\mathrm{j}}, \mathrm{x}_{\mathrm{j}, 1}, \mathrm{x}_{\mathrm{j}, 2}, \mathrm{c}_{\mathrm{j}}\right)$ from $\mathrm{K}^{\text {list. }}$.

2. Pick $\mathrm{h} \stackrel{\$}{\leftarrow}\{0,1\}^{l_{0}}$ and $\pi \stackrel{\$}{\leftarrow}\{0,1\}^{l_{1}}$; compute $\mathrm{v}=\mathrm{H}_{1}(\mathrm{~h}, \pi), \mathrm{V}=\mathrm{g}^{\mathrm{v}}$ and $\mathrm{W}=\mathrm{H}_{3}\left(\mathrm{pk}_{\mathrm{j}, 2}^{\mathrm{v}}\right)$ $\oplus(\mathrm{h} \| \pi)$.

3. Construct the first component $\mathrm{rk}_{\mathrm{i} \rightarrow \mathrm{j}}^{(1)}$ according to the following cases:

- $c_{\mathrm{i}}=1$ or $\mathrm{c}_{\mathrm{i}}=$ '-': Define $\mathrm{rk}_{\mathrm{i} \rightarrow \mathrm{j}}^{(1)}=\frac{\mathrm{h}}{\mathrm{x}_{\mathrm{i}, 1} \mathrm{H}_{5}\left(\mathrm{pk}_{\mathrm{i}, 2}\right)+\mathrm{x}_{\mathrm{i}, 2}}$.

- $\left(\mathrm{c}_{\mathrm{i}}=0 \wedge \mathrm{c}_{\mathrm{j}}=1\right)$ or $\left(\mathrm{c}_{\mathrm{i}}=0 \wedge \mathrm{c}_{\mathrm{j}}=0\right)$ : Pick $\mathrm{rk}_{\mathrm{i} \rightarrow \mathrm{j}}^{(1)} \stackrel{\$}{\leftarrow} \mathrm{Z}_{\mathrm{q}}^{*}$.

- $\quad\left(c_{i}=0 \wedge c_{j}=\right.$ '-'): Output "failure" and abort.

4. If $\mathcal{B}$ does not abort, add $\left(\mathrm{pk}_{\mathrm{i}}, \mathrm{pk}_{\mathrm{j}},\left(\mathrm{rk}_{\mathrm{i} \rightarrow \mathrm{j}}^{(1)}, \mathrm{V}, \mathrm{W}\right), \mathrm{h}\right)$ into list $\mathrm{R}^{\text {list }}$, return $\left(\mathrm{rk}_{\mathrm{i} \rightarrow \mathrm{j}}^{(1)}, \mathrm{V}, \mathrm{W}\right)$.

- Condition key query $\left(\mathrm{pk}_{\mathrm{i}}, \mathrm{w}\right)$. If $\mathrm{C}^{\text {list }}$ has an entry for $\left(\mathrm{pk}_{\mathrm{i}}, \mathrm{w}\right)$, return the predefined condition key $\mathrm{ck}_{\mathrm{i}, \mathrm{w}}$ to $\mathcal{A}$. Otherwise algorithm $\mathscr{B}$ acts as follows:

1. Recover tuples $\left(\mathrm{pk}_{\mathrm{i}}, \mathrm{x}_{\mathrm{i}, 1}, \mathrm{x}_{\mathrm{i}, 2}, \mathrm{c}_{\mathrm{i}}\right)$ from $\mathrm{K}^{\text {list }}$ and $\left(\mathrm{w}, \mathrm{pk}_{\mathrm{i}}, \mathrm{t}, \mathrm{S}\right)$ from $\mathrm{H}_{6}^{\text {list }}$.

2. It constructs the condition key $\mathrm{ck}_{\mathrm{i}, \mathrm{w}}$ for adversary $\mathcal{A}$ according to the following cases:

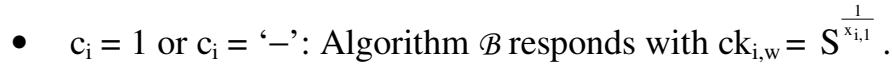

- $\quad c_{i}=0$ : Algorithm $B$ responds with $\mathrm{ck}_{\mathrm{i}, \mathrm{w}}=\left(\mathrm{g}^{\mathrm{a}}\right)^{\frac{1}{\mathrm{x}_{\mathrm{i}, 1}}}$ which is same as $\mathrm{S}^{\frac{1}{\mathrm{k}_{\mathrm{i}, 1}}}$.

3. Add $\left(\mathrm{pk}_{\mathrm{i}}, \mathrm{w}, \mathrm{ck}_{\mathrm{i}, \mathrm{w}}\right)$ to $\mathrm{C}^{\text {list }}$.

- Re-encryption query $\left.\left(\mathrm{pk}_{\mathrm{i}}, \mathrm{pk}_{\mathrm{j}}, \mathrm{w}, \zeta_{\mathrm{i}}\right)\right\}$. Algorithm $B$ parses $\zeta_{\mathrm{i}}=(\mathrm{A}, \mathrm{B}, \mathrm{C}, \mathrm{D}, \mathrm{E})$. Return $\perp$ if Validity() returns $\perp$. Otherwise it constructs the condition key $\mathrm{ck}_{\mathrm{i}, \mathrm{w}}$ by issuing a condition key query $\left(\mathrm{pk}_{\mathrm{i}}, \mathrm{w}\right)$ and does the following:

1. Recover tuples $\left(\mathrm{pk}_{\mathrm{i}}, \mathrm{x}_{\mathrm{i}, 1}, \mathrm{x}_{\mathrm{i}, 2}, \mathrm{c}_{\mathrm{i}}\right)$ and $\left(\mathrm{pk}_{\mathrm{j}}, \mathrm{x}_{\mathrm{j}, 1}, \mathrm{x}_{\mathrm{j}, 2}, \mathrm{c}_{\mathrm{j}}\right)$ from $\mathrm{K}^{\text {list }}$.

2. If ( $\left.\mathrm{c}_{\mathrm{i}}=0 \wedge \mathrm{c}_{\mathrm{j}}={ }^{\prime}-{ }^{\prime}\right)$ ) does not hold, issue a re-encryption key generation query ( $\mathrm{pk}_{\mathrm{i}}$, $\left.\mathrm{pk}_{\mathrm{j}}\right)$ to obtain $\mathrm{rk}_{\mathrm{i} \rightarrow \mathrm{j}}$, and then $\operatorname{ReEncrypt}\left(\mathrm{rk}_{\mathrm{i} \rightarrow \mathrm{j}}, \mathrm{ck}_{\mathrm{i}, \mathrm{w}}, \zeta_{\mathrm{i}}, \mathrm{pk}_{\mathrm{i}}, \mathrm{pk}_{\mathrm{j}}\right)$ to $\mathcal{A}$.

3. Else $\mathfrak{B}$ does the following.

- Pick $\mathrm{h} \stackrel{\$}{\leftarrow}\{0,1\}^{l_{0}}$ and $\pi \stackrel{\$}{\leftarrow}\{0,1\}^{l_{1}}$ and compute $\mathrm{v}=\mathrm{H}_{1}(\mathrm{~h}, \pi)$.

- Compute $\mathrm{V}=\mathrm{g}^{\mathrm{v}}$ and $\mathrm{W}=\mathrm{H}_{3}\left(\mathrm{pk}_{\mathrm{j}, 2}^{\mathrm{v}}\right) \oplus(\mathrm{h} \| \pi)$.

- Since the ciphertext is valid, issue a decryption query $\left(\mathrm{pk}_{\mathrm{i}}, \zeta_{\mathrm{i}}\right)$ and get message $\mathrm{m}$.

- Pick $\mathrm{r}^{\prime} \stackrel{\$}{\leftarrow}\{0,1\}^{l_{1}}$. Compute $\mathrm{r}=\mathrm{H}_{2}\left(\mathrm{~m}, \mathrm{r}^{\prime}, \mathrm{pk}_{\mathrm{i}}, \mathrm{w}\right)$.

- Compute $\mathrm{A}^{\prime}=\mathrm{g}^{\mathrm{rh}}$ and $\mathrm{C}^{\prime}=\mathrm{H}_{3}\left(\mathrm{~g}^{\mathrm{r}}\right) \oplus\left(\mathrm{m} \| \mathrm{r}^{\prime}\right)$. 
International Journal of Network Security \& Its Applications (IJNSA), Vol.4, No.2, March 2012

- Add tuple (h, $\pi, v)$ to $\mathrm{H}_{\mathrm{i}}^{\text {list }}$ and tuple $\left(\mathrm{m}, \mathrm{r}^{\prime}, \mathrm{pk}_{\mathrm{i}}, \mathrm{w}, \mathrm{r}\right)$ to $\mathrm{H}_{2}^{\text {list }}$, if they are not present in their respective lists.

- $\operatorname{Return}\left(\mathrm{A}^{\prime}, \mathrm{C}^{\prime}, \mathrm{V}, \mathrm{W}\right)$ to $\mathrm{A}$ as the re-encrypted ciphertext.

- Decryption query $\left(\mathrm{pk}_{\mathrm{i}}, \mathrm{w}^{\prime}, \zeta_{\mathrm{i}}\right)$ or $\left(\mathrm{pk}_{\mathrm{i}}, \zeta_{\mathrm{i}}\right)$. B recovers tuple $\left(\mathrm{pk}_{\mathrm{i}}, \mathrm{x}_{\mathrm{i}, 1}, \mathrm{x}_{\mathrm{i}, 2}, \mathrm{c}\right)$ from $\mathrm{K}^{\text {list }}$. If $\mathrm{c}=$ 1 or $\mathrm{c}=$ '-', algorithm $\mathcal{B}$ runs $\left.\operatorname{Decrypt}\left(\left(\mathrm{x}_{\mathrm{i}, 1}, \mathrm{x}_{\mathrm{i}, 2}\right), \zeta_{\mathrm{i}}\right)\right)$ and returns the result to $\mathcal{A}$. Otherwise, algorithm $\mathcal{B}$ works according to the following two cases:

- $\zeta_{\mathrm{i}}$ is an original ciphertext $\zeta_{\mathrm{I}}=(\mathrm{A}, \mathrm{B}, \mathrm{C}, \mathrm{D}, \mathrm{E})$ :

1. Return $\perp$ if Validity() returns $\perp$.

2. Construct condition key $\mathrm{ck}_{\mathrm{i}, \mathrm{w}^{\prime}}$ as in the condition key query and define $\mathrm{C}=\mathrm{C}^{\prime} \oplus$ $\mathrm{H}_{7}\left(\hat{\mathrm{e}}\left(\mathrm{B}, \mathrm{ck}_{\mathrm{i}, \mathrm{w}^{\prime}}\right)\right)$.

3. Search tuples $\left(\mathrm{m}, \mathrm{r}^{\prime}, \mathrm{pk}, \mathrm{w}, \mathrm{r}\right) \in \mathrm{H}_{2}^{\text {list }}$ and $(\mathrm{R}, \beta) \in \mathrm{H}_{3}^{\text {list }}$ such that $\mathrm{pk}_{\mathrm{i}}=\mathrm{pk}, \mathrm{w}=$ $\mathrm{w}^{\prime}, \beta \oplus\left(\mathrm{m} \| \mathrm{r}^{\prime}\right)=\mathrm{C}, \mathrm{g}^{\mathrm{r}}=\mathrm{R}$ and $\left(\mathrm{pk}_{\mathrm{i}, 1}^{\mathrm{H}_{5}\left(\mathrm{pk}_{\mathrm{i}, 2}\right)} \mathrm{pk}_{\mathrm{i}, 2}\right)^{\mathrm{r}}=\mathrm{A}$.

4. If yes, return $\mathrm{m}$ to $\mathcal{A}$. Otherwise, return $\perp$.

- $\zeta_{\mathrm{i}}$ is a re-encrypted ciphertext $\zeta_{\mathrm{i}}=\left(\mathrm{A}^{\prime}, \mathrm{C}^{\prime}, \mathrm{V}, \mathrm{W}\right)$ :

1. Search tuples $\left(m, r^{\prime}, p k, w, r\right) \in H_{2}^{\text {list }},(h, \pi, v) \in H_{1}^{\text {list }},(R, \beta) \in H_{3}^{\text {list }}$ and $\left(R^{\prime}\right.$, $\left.\beta^{\prime}\right) \in \mathrm{H}_{3}^{\text {list }}$ such that $\mathrm{pk}_{\mathrm{i}}=\mathrm{pk}, \mathrm{w}=\mathrm{w}^{\prime}, \mathrm{g}^{\mathrm{rh}}=\mathrm{A}^{\prime}, \mathrm{g}^{\mathrm{r}}=\mathrm{R}, \beta \oplus\left(\mathrm{m} \| \mathrm{r}^{\prime}\right)=\mathrm{C}^{\prime}, \mathrm{g}^{\mathrm{v}}=\mathrm{V}$, $\beta^{\prime} \oplus(\mathrm{h} \| \pi)=\mathrm{W}$ and $\mathrm{pk}_{\mathrm{i}, 2}^{\mathrm{v}}=\mathrm{R}^{\prime}$.

2. If yes, return $m$ to $\mathcal{A}$. Otherwise, return $\perp$.

Challenge. When $\mathcal{A}$ decides that Phase 1 is over, it outputs a public key $\mathrm{pk}_{\mathrm{i}^{*}}=\left(\mathrm{pk}_{\mathrm{i}^{*}, 1}, \mathrm{pk}_{\mathrm{i}^{*}, 2}\right)$, a condition $\mathrm{w}^{*}$ and two equal-length messages $\mathrm{m}_{0}, \mathrm{~m}_{1} \in\{0,1\}^{l_{0}+l_{1}}$. Algorithm $\mathscr{B}$ responds as follows:

1. Recover tuple $\left(\mathrm{pk}_{\mathrm{i}^{*},}, \mathrm{x}_{\mathrm{i}^{*}, 1}, \mathrm{x}_{\mathrm{i}^{*}, 2}, \mathrm{c}^{*}\right)$ from $\mathrm{K}^{\text {list }}$. If $\mathrm{c}^{*} \neq 0, \mathcal{B}$ outputs "failure" and aborts. Otherwise, $\mathcal{B}$ proceeds to execute the following steps.

2. Pick $\mathrm{s}^{*}, \mathrm{z}^{*} \stackrel{\$}{\leftarrow} \mathrm{Z}_{\mathrm{q}}^{*}$ and compute $\mathrm{B}^{*}=\left(\mathrm{g}^{\frac{1}{a}}\right)^{\mathrm{s}^{*} \mathrm{x}^{*}, 1}$ and $\mathrm{D}^{*}=\left(\mathrm{g}^{\frac{1}{a}}\right)^{\mathrm{Z}^{*} \mathrm{x}^{*}, 1}$.

3. Pick $\mathrm{C}^{*} \stackrel{\$}{\leftarrow}\{0,1\}^{l_{0}+l_{1}}$.

4. Compute $\mathrm{A}^{*}=\left(\mathrm{g}^{\mathrm{b}}\right)^{\mathrm{x}_{\mathrm{i}, 1} \mathrm{H}_{5}\left(\mathrm{pk}_{\mathrm{i}^{*}, 2}\right)+\mathrm{x}_{\mathrm{i}^{*}, 2}}$ and $\mathrm{E}^{*}=\mathrm{s}^{*}+\mathrm{z}^{*} \mathrm{H}_{4}\left(\mathrm{~A}^{*}, \mathrm{~B}^{*}, \mathrm{C}^{*}, \mathrm{D}^{*}\right) \bmod \mathrm{q}$.

5. Construct the condition key $\mathrm{ck}_{\mathrm{i}^{*}, \mathrm{w}^{*}}$, as in the condition key query.

6. Pick a random bit $\delta \stackrel{\$}{\leftarrow}\{0,1\}$ and $\mathrm{r}^{\prime} \stackrel{\$}{\leftarrow}\{0,1\}^{l_{1}}$. Implicitly define $\mathrm{H}_{2}\left(\mathrm{~m}_{\delta}, \mathrm{r}^{\prime}, \mathrm{pk}_{\mathrm{i}^{*}}, \mathrm{w}^{*}\right)=\mathrm{ab}$ and

$\mathrm{H}_{3}\left(\mathrm{~g}^{\mathrm{ab}}\right)=\mathrm{C}^{*} \oplus\left(\mathrm{m}_{\delta} \| \mathrm{r}^{\prime}\right) \oplus \mathrm{H}_{7}\left(\hat{\mathrm{e}}\left(\mathrm{B}^{*}, \mathrm{ck}_{\mathrm{i}^{*}, \mathrm{w}^{*}}\right)\right)$ (note that $\mathscr{B}$ knows neither ab nor $\left.\mathrm{g}^{\mathrm{ab}}\right)$.

7. Return $\zeta^{*}=\left(\mathrm{A}^{*}, \mathrm{~B}^{*}, \mathrm{C}^{*}, \mathrm{D}^{*}, \mathrm{E}^{*}\right)$ as the challenged ciphertext to adversary $\mathrm{A}$.

Observe that the challenge ciphertext $\zeta^{*}$ is identically distributed as the real one from the construction. To see this, letting $r^{*}=a b$, we have

$$
\begin{aligned}
\mathrm{A}^{*} & =\left(\mathrm{g}^{\mathrm{b}}\right)^{\mathrm{x}_{\mathrm{i}^{*}, 1} \mathrm{H}_{5}\left(\mathrm{pk}_{\mathrm{i}^{*}, 2}\right)+\mathrm{x}_{\mathrm{i}^{*}, 2}}=\left(\left(\mathrm{g}^{\frac{1}{a}}\right)^{\mathrm{x}_{\mathrm{i}^{*}, 1} \mathrm{H}_{5}\left(\mathrm{pk}_{\mathrm{i}^{*}, 2}\right)+\mathrm{x}_{\mathrm{i}^{*}, 2}}\right)^{\mathrm{ab}} \\
& =\left(\mathrm{pk}_{\mathrm{i}^{*}, 1}^{\mathrm{H}_{5}\left(\mathrm{pk}_{\mathrm{i}^{*}, 2}\right)} \mathrm{pk}_{\mathrm{i}^{*}, 2}\right)^{\mathrm{r}^{*}} \\
\mathrm{~B}^{*} & =\left(\left(\mathrm{g}^{\frac{1}{a}}\right)^{*^{*} \mathrm{x}_{\mathrm{i}^{*}, 1}}=\mathrm{pk}_{\mathrm{i}^{*}, 1}^{\mathrm{s}^{*}}\right. \\
\mathrm{C}^{*} & =\mathrm{H}_{3}\left(\mathrm{~g}^{\mathrm{ab}}\right) \oplus\left(\mathrm{m}_{\delta} \| \mathrm{r}^{\prime}\right) \oplus \mathrm{H}_{7}\left(\hat{\mathrm{e}}\left(\mathrm{B}^{*}, \mathrm{ck}_{\mathrm{i}^{*}, \mathrm{w}^{*}}\right)\right) \\
& =\mathrm{H}_{3}\left(\mathrm{~g}^{\mathrm{ab}}\right) \oplus\left(\mathrm{m}_{\delta} \| \mathrm{r}^{\prime}\right) \oplus \mathrm{H}_{7}\left(\hat{\mathrm{e}}\left(\left(\mathrm{g}^{\frac{1}{a}}\right)^{s^{*} \mathrm{i}_{\mathrm{i}^{*}, 1}},\left(\mathrm{~g}^{\mathrm{a}}\right)^{\frac{1}{\mathrm{x}^{* * 1}, 1}}\right)\right)
\end{aligned}
$$


International Journal of Network Security \& Its Applications (IJNSA), Vol.4, No.2, March 2012

$=\mathrm{H}_{3}\left(\mathrm{~g}^{\mathrm{ab}}\right) \oplus\left(\mathrm{m}_{\delta} \| \mathrm{r}^{\prime}\right) \oplus \mathrm{H}_{7}\left(\hat{\mathrm{e}}\left(\mathrm{g}, \mathrm{g}^{\mathrm{y}}\right)^{\mathrm{s}^{*}}\right)$

$=\mathrm{H}_{3}\left(\mathrm{~g}^{\mathrm{r}^{*}}\right) \oplus\left(\mathrm{m}_{\delta} \| \mathrm{r}^{\prime}\right) \oplus \mathrm{H}_{7}\left(\hat{\mathrm{e}}\left(\mathrm{g}, \mathrm{H}_{6}\left(\mathrm{w}^{*}, \mathrm{pk}_{\mathrm{i}^{*}, \mathrm{w}^{*}}\right)\right)^{\mathrm{s}^{*}}\right)$

$\mathrm{D}^{*}=\left(\mathrm{g}^{\frac{1}{\mathrm{a}}}\right)^{\mathrm{z}^{*} \mathrm{x}_{\mathrm{i}^{*}, 1}}=\mathrm{pk}_{\mathrm{i}^{*}, 1}^{\mathrm{z}^{*}}$

$\mathrm{E}^{*}=\mathrm{s}^{*}+\mathrm{z}^{*} \mathrm{H}_{4}\left(\mathrm{~A}^{*}, \mathrm{~B}^{*}, \mathrm{C}^{*}, \mathrm{D}^{*}\right) \bmod \mathrm{q}$

Phase 2. Adversary $\mathcal{A}$ continues to issue queries as in Phase 1 , with the restrictions prescribed in the IND-CPRE-CCA game. Algorithm $B$ responds to these queries for $\mathcal{A}$ as in Phase 1.

Guess. Eventually, adversary $\mathcal{A}$ returns a guess $\delta^{\prime} \in\{0,1\} \mathscr{B}$. Algorithm $\mathscr{B}$ randomly picks a tuple $(\mathrm{R}, \beta)$ from the $\mathrm{H}_{3}^{\text {list }}$ and outputs $\mathrm{R}$ as the solution to the given problem instance.

Analysis. Now let's analyse the simulation. From the constructions of $\mathrm{H}_{1}, \mathrm{H}_{5}, \mathrm{H}_{6}$ and $\mathrm{H}_{7}$, it is clear that the simulations of these oracles are perfect. Let $\mathrm{AskH}_{4}^{*}$ be the event that $\mathrm{A}$ queried $\left(\mathrm{A}^{*}, \mathrm{~B}^{*}, \mathrm{C}^{*}, \mathrm{D}^{*}\right)$ to $\mathrm{H}_{4}$ before challenge phase. The simulation of $\mathrm{H}_{4}$ is perfect as long as $\mathrm{AskH}_{4}^{*}$ did not occur. Since $\mathrm{C}^{*}$ is randomly chosen from $\{0,1\}^{l_{0}+l_{1}}$ by the challenger in the challenge phase, we have $\operatorname{Pr}\left[\mathrm{AskH}_{4}^{*}\right]=\frac{\mathrm{q}_{\mathrm{H}_{4}}}{2^{l_{0}+l_{1}}}$. Let $\mathrm{AskH}_{2}^{*}$ be the event that $\left(\mathrm{m}_{\delta}, \mathrm{r}^{\prime}, \mathrm{pk}_{\mathrm{i}^{*}}, \mathrm{w}^{*}\right)$ has been queried to $\mathrm{H}_{2}$ and $\mathrm{AskH}_{3}^{*}$ be the event that $\mathrm{g}^{\mathrm{ab}}$ has been queried to $\mathrm{H}_{3}$. The simulations of $\mathrm{H}_{2}$ and $\mathrm{H}_{3}$ are perfect as long as $\mathrm{AskH}_{2}^{*}$ and $\mathrm{AskH}_{3}^{*}$ did not occur, where $\delta$ and $\mathrm{r}^{\prime}$ are chosen by $\mathcal{B}$ in the challenge phase.

$\mathscr{B}^{\prime}$ 's responses to $\mathcal{A}$ 's uncorrupted/corrupted key generation queries are perfect. Let Abort denote the event of $\mathscr{B}$ 's aborting during the simulation of the re-encryption key queries or in the challenge phase. We have $\operatorname{Pr}[\neg$ Abort $] \geq \theta^{\mathrm{q}_{\mathrm{rk}}}(1-\theta)$, which is maximized at $\theta_{\mathrm{opt}}=\frac{\mathrm{q}_{\mathrm{rk}}}{1+\mathrm{q}_{\mathrm{rk}}}$. Using $\theta_{\mathrm{opt}}$, the probability $\operatorname{Pr}[\neg$ Abort $]$ is at least $\frac{1}{\mathrm{e}\left(1+\mathrm{q}_{\mathrm{rk}}\right)}$.

The simulation of the re-encryption key queries is same as the real one, except for the case $\left(c_{i}=\right.$ $\left.0 \wedge c_{j}=1\right)$ or $\left(c_{i}=0 \wedge c_{j}=0\right)$, in which the component $r_{\mathrm{i} \rightarrow j}^{(1)}$ is randomly chosen. If Abort does not happen, this is computationally indistinguishable from the real world because:

1. Secret key $\mathrm{sk}_{\mathrm{j}}$ is unknown to $\mathcal{A}$ since $\mathrm{c}_{\mathrm{j}} \neq$ ' - '.

2. $\mathrm{h}$ is encrypted under $\mathrm{pk}_{\mathrm{j}}$ using the "hashed" ElGamal encryption scheme. So, if $\mathcal{A}$ can distinguish $\mathrm{rk}_{\mathrm{i} \rightarrow \mathrm{j}}$ from $\mathrm{rk}_{\mathrm{i} \rightarrow \mathrm{j}}^{\prime}$, it means that $\mathcal{A}$ can determine $(\mathrm{V}, \mathrm{W})$ is an encryption of $\mathrm{h}$ or $\mathrm{h}^{\prime}$, which breaks the CCA security of the "hashed" ElGamal based on the CDH assumption.

The re-encryption queries are also perfect, unless $\mathcal{A}$ can submit valid original ciphertexts without querying $\mathrm{H}_{2}$ or $\mathrm{H}_{3}$ (denote this event by REErr). This is because we issue a decryption query in the third case of the re-encryption query. We will calculate Pr[REErr] shortly.

The simulation of the decryption oracle is perfect, with the exception that simulation errors may occur in rejecting some valid ciphertexts. $\mathcal{A}$ can submit valid original ciphertexts without querying $\mathrm{H}_{2}$ or $\mathrm{H}_{3}$ (denote this event by DErr). Let Valid be the event that the ciphertext is 
International Journal of Network Security \& Its Applications (IJNSA), Vol.4, No.2, March 2012

valid. Let $\mathrm{AskH}_{3}$ and $\mathrm{AskH}_{2}$ be the events $\mathrm{g}^{\mathrm{r}}$ has been queried to $\mathrm{H}_{3}$ and $\left(\mathrm{m}, \mathrm{r}^{\prime}, \mathrm{w}\right)$ has been queried to $\mathrm{H}_{2}$ respectively. We have,

$$
\begin{aligned}
\operatorname{Pr}\left[\text { Valid } \mid \neg \text { AskH }_{2}\right] & =\operatorname{Pr}\left[\text { Valid } \wedge \text { AshH }_{3} \mid \neg \text { AskH }_{2}\right]+\operatorname{Pr}\left[\text { Valid } \wedge \neg \text { AskH }_{3} \mid \neg \text { AskH }_{2}\right] \\
& \leq \operatorname{Pr}\left[\text { AskH }_{3} \mid \neg \text { AskH }_{2}\right]+\operatorname{Pr}\left[\text { Valid } I \neg \text { AskH }_{3} \wedge \neg \text { AskH }_{2}\right] \\
& \leq \frac{\mathrm{q}_{\mathrm{H}_{3}}}{2^{1^{+}+l_{1}}}+\frac{1}{q}
\end{aligned}
$$

Similarly, we have $\operatorname{Pr}\left[\right.$ Valid $\left.I \neg \mathrm{AskH}_{3}\right] \leq \frac{\mathrm{q}_{\mathrm{H}_{2}}}{2^{1_{0}+1_{1}}}+\frac{1}{\mathrm{q}}$. Thus we have,

$\operatorname{Pr}\left[\right.$ Valid I $\left.\left(\neg \mathrm{AskH}_{2} \vee \mathrm{AskH}_{3}\right)\right] \leq \operatorname{Pr}\left[\right.$ Valid $\left.I \neg \mathrm{AskH}_{2}\right]+\operatorname{Pr}\left[\right.$ Valid I $\left.\neg \mathrm{AskH}_{3}\right]$

$$
\leq \frac{\mathrm{q}_{\mathrm{H}_{2}}+\mathrm{q}_{\mathrm{H}_{3}}}{2^{\mathrm{I}_{0}+1_{1}}}+\frac{2}{\mathrm{q}}
$$

Let DErr be the event that Valid I( $\left.\neg \mathrm{AskH}_{2} \vee \neg \mathrm{AskH}_{3}\right)$ happens during the entire simulation.

Then since $A$ issues utmost $q_{d}$ decryption oracles, we have $\operatorname{Pr}[D E r r] \leq \frac{\left(\mathrm{q}_{\mathrm{H}_{2}}+\mathrm{q}_{\mathrm{H}_{3}}\right) \mathrm{q}_{\mathrm{d}}}{2^{1_{0}+1_{1}}}+\frac{2 \mathrm{q}_{\mathrm{d}}}{\mathrm{q}}$.

By the definition of REErr as stated above, since $\mathcal{A}$ issues utmost qre re-encryption oracles, we have

$\operatorname{Pr}[$ REErr $] \leq \frac{\left(\mathrm{q}_{\mathrm{H}_{2}}+\mathrm{q}_{\mathrm{H}_{3}}\right) \mathrm{q}_{\mathrm{re}}}{2^{\mathrm{l}_{0}+1_{1}}}+\frac{2 \mathrm{q}_{\mathrm{re}}}{\mathrm{q}}$

Now, let Good denote the event

$$
\left(\mathrm{AskH}_{3}^{*} \vee\left(\mathrm{AskH}_{2}^{*} \mid \neg \mathrm{AskH}_{3}^{*}\right) \vee \mathrm{AskH}_{4}^{*} \vee \mathrm{REErr} \vee \text { DErr }\right) \mid \neg \text { Abort . }
$$

If Good does not happen, due to the randomness of the output of the random oracle $\mathrm{H}_{3}$, it is clear that $\mathcal{A}$ cannot gain any advantage greater than $\frac{1}{2}$ in guessing $\delta$. Thus we have $\operatorname{Pr}\left[\delta^{\prime}=\delta \mid \neg\right.$ Good $]=\frac{1}{2}$. Hence by splitting $\operatorname{Pr}\left[\delta^{\prime}=\delta\right]$, we have

$$
\begin{aligned}
\operatorname{Pr}\left[\delta^{\prime}=\delta\right]= & \operatorname{Pr}\left[\delta^{\prime}=\delta \mid \neg \text { Good }\right] \operatorname{Pr}[\neg \text { Good }]+\operatorname{Pr}\left[\delta^{\prime}=\delta \mid \text { Good }\right] \operatorname{Pr}[\text { Good }] \\
& \leq \frac{1}{2} \operatorname{Pr}[\neg \text { Good }]+\operatorname{Pr}[\text { Good }] \\
& \leq \frac{1}{2}+\frac{1}{2} \operatorname{Pr}[\text { Good }] \\
\operatorname{Pr}\left[\delta^{\prime}=\delta\right] & \geq \operatorname{Pr}\left[\delta^{\prime}=\delta \mid \neg \text { Good }\right] \operatorname{Pr}[\neg \text { Good }] \\
& =\frac{1}{2}-\frac{1}{2} \operatorname{Pr}[\text { Good }]
\end{aligned}
$$

By definition of the advantage for the IND-CPRE-CCA adversary, we then have

$$
\begin{aligned}
& \varepsilon-\psi=\left|2 \times \operatorname{Pr}\left[\delta^{\prime}=\delta\right]-1\right| \\
& \leq \operatorname{Pr} \text { [Good] } \\
& =\operatorname{Pr}\left[\left(\text { AskH }_{3}^{*} \vee\left(\text { AskH }_{2}^{*} \mid \neg \text { AskH }_{3}^{*}\right) \vee \text { AskH }_{4}^{*} \vee \text { REErr } \vee \text { DErr }\right) \mid \neg \text { Abort }\right] \\
& =\frac{\operatorname{Pr}\left[\left(\text { AskH }_{3}^{*}\right]+\operatorname{Pr}\left[\text { AskH }_{2}^{*} \mid \neg \text { AskH }_{3}^{*}\right]+\operatorname{Pr}\left[\text { AskH }_{4}^{*}\right]\right.}{\operatorname{Pr}[\neg \text { Abort }]}+\frac{\operatorname{Pr}[\text { REErr }]+\operatorname{Pr}[\text { DErr }]}{\operatorname{Pr}[\neg \text { Abort }]} \text {. }
\end{aligned}
$$

Substituting values which have been computed, we get 
International Journal of Network Security \& Its Applications (IJNSA), Vol.4, No.2, March 2012

$\operatorname{Pr}\left[\mathrm{AskH}_{3}^{*}\right] \geq \operatorname{Pr}[\neg$ Abort $] \cdot(\varepsilon-\psi)-\operatorname{Pr}\left[\mathrm{AskH}_{2}^{*} \mid \neg \mathrm{AskH}_{3}^{*}\right]-\operatorname{Pr}\left[\mathrm{AskH}_{4}^{*}\right]-\operatorname{Pr}[$ Reerr $]-\operatorname{Pr}[\mathrm{Derr}]$

$$
\begin{aligned}
& \geq \frac{\varepsilon-\psi}{\varepsilon\left(1+\mathrm{q}_{\mathrm{rk}}\right)}-\frac{\mathrm{q}_{\mathrm{H}_{2}}}{2^{1_{0}+\mathrm{l}_{1}}}-\frac{\mathrm{q}_{\mathrm{H}_{4}}}{2^{1_{0}+1_{1}}}-\frac{\left(\mathrm{q}_{\mathrm{H}_{2}}+\mathrm{q}_{\mathrm{H}_{3}}\right) \mathrm{q}_{\mathrm{re}}}{2^{1_{0}+1_{1}}}-\frac{2 \mathrm{q}_{\mathrm{re}}}{\mathrm{q}}-\frac{\left(\mathrm{q}_{\mathrm{H}_{2}}+\mathrm{q}_{\mathrm{H}_{3}}\right) \mathrm{q}_{\mathrm{d}}}{2^{1_{0}+1_{1}}}-\frac{2 \mathrm{q}_{\mathrm{d}}}{\mathrm{q}} \\
& =\frac{\varepsilon-\psi}{\varepsilon\left(1+\mathrm{q}_{\mathrm{rk}}\right)}-\frac{\mathrm{q}_{\mathrm{H}_{2}}+\mathrm{q}_{\mathrm{H}_{4}}+\left(\mathrm{q}_{\mathrm{H}_{2}}+\mathrm{q}_{\mathrm{H}_{3}}\right)\left(\mathrm{q}_{\mathrm{re}}+\mathrm{q}_{\mathrm{d}}\right)}{2^{\mathrm{1}_{0}+\mathrm{l}_{1}}}-\frac{2\left(\mathrm{q}_{\mathrm{re}}+\mathrm{q}_{\mathrm{d}}\right)}{\mathrm{q}}
\end{aligned}
$$

If $\mathrm{AskH}_{3}^{*}$ happens, algorithm $\mathscr{B}$ will be able to solve $\mathrm{mCDH}$ instance. Therefore we get,

$$
\begin{aligned}
\varepsilon^{\prime} & \geq \frac{1}{\mathrm{q}_{\mathrm{H}_{3}}} \operatorname{Pr}\left[\text { AskH }_{3}^{*}\right] \\
& \geq \frac{1}{\mathrm{q}_{\mathrm{H}_{3}}}\left(\frac{\varepsilon-\psi}{\varepsilon\left(1+\mathrm{q}_{\mathrm{rk}}\right)}-\frac{\mathrm{q}_{\mathrm{H}_{2}}+\mathrm{q}_{\mathrm{H}_{4}}+\left(\mathrm{q}_{\mathrm{H}_{2}}+\mathrm{q}_{\mathrm{H}_{3}}\right)\left(\mathrm{q}_{\mathrm{re}}+\mathrm{q}_{\mathrm{d}}\right)}{2^{\mathrm{1}_{0}+\mathrm{l}_{1}}}-\frac{2\left(\mathrm{q}_{\mathrm{re}}+\mathrm{q}_{\mathrm{d}}\right)}{\mathrm{q}}\right)
\end{aligned}
$$

From the description of the simulation, $\mathfrak{B}^{\prime}$ s running time can be bounded by

$$
\begin{aligned}
\mathrm{t}^{\prime} \leq \mathrm{t} & +\left(\mathrm{q}_{\mathrm{H}_{1}}+\mathrm{q}_{\mathrm{H}_{2}}+\mathrm{q}_{\mathrm{H}_{3}}+\mathrm{q}_{\mathrm{H}_{4}}+\mathrm{q}_{\mathrm{H}_{5}}+\mathrm{q}_{\mathrm{H}_{6}}+\mathrm{q}_{\mathrm{H}_{7}}+\mathrm{q}_{\mathrm{u}}+\mathrm{q}_{\mathrm{c}}+\mathrm{q}_{\mathrm{rk}}+\mathrm{q}_{\mathrm{ck}}+\mathrm{q}_{\mathrm{re}}+\mathrm{q}_{\mathrm{d}}\right) O(1) \\
& +\left(2 \mathrm{q}_{\mathrm{c}}+2 \mathrm{q}_{\mathrm{u}}+6 \mathrm{q}_{\mathrm{rk}}+\mathrm{q}_{\mathrm{ck}}+\left(\mathrm{q}_{\mathrm{re}}+1\right)\left(2 \mathrm{q}_{\mathrm{d}}+\left(2 \mathrm{q}_{\mathrm{H}_{2}}+2 \mathrm{q}_{\mathrm{H}_{3}}\right) \mathrm{q}_{\mathrm{d}}\right)\right) \mathrm{t}_{\exp }+\left(\mathrm{q}_{\mathrm{re}}+\mathrm{q}_{\mathrm{d}}\right) \mathrm{t}_{\mathrm{p}}
\end{aligned}
$$

This completes the proof of Theorem 1 .

Theorem 2. Our scheme is IND-CPRE-CCA secure in the random oracle model, assuming the mCBDH assumption holds in groups $G, G_{T}$ and the Schnorr signature is EUF-CMA secure. Concretely, if there exists a Type II adversary $\mathcal{A}$, who asks at most $\mathrm{q}_{\mathrm{H}_{\mathrm{i}}}$ random oracle queries to $\mathrm{H}_{\mathrm{i}}$ with $\mathrm{i} \in\{1,2, \ldots, 7\}$, and breaks the $\left(\mathrm{t}, \mathrm{q}_{\mathrm{u}}, \mathrm{q}_{\mathrm{c}}, \mathrm{q}_{\mathrm{rk}}, \mathrm{q}_{\mathrm{ck}}, \mathrm{q}_{\mathrm{re}}, \mathrm{q}_{\mathrm{d}}, \varepsilon\right.$ )-IND-CPRE-CCA of our scheme, then, for any $0<\psi<\varepsilon$, there exists

1. either an algorithm $\mathscr{B}$ which can break the $\left(\mathrm{t}^{\prime}, \varepsilon^{\prime}\right)-\mathrm{mCBDH}$ assumption in $\mathrm{G}$ with

$$
\begin{gathered}
\mathrm{t}^{\prime} \leq \mathrm{t}+\left(\mathrm{q}_{\mathrm{H}_{1}}+\mathrm{q}_{\mathrm{H}_{2}}+\mathrm{q}_{\mathrm{H}_{3}}+\mathrm{q}_{\mathrm{H}_{4}}+\mathrm{q}_{\mathrm{H}_{5}}+\mathrm{q}_{\mathrm{H}_{6}}+\mathrm{q}_{\mathrm{H}_{7}}+\mathrm{q}_{\mathrm{u}}+\mathrm{q}_{\mathrm{c}}+\mathrm{q}_{\mathrm{rk}}+\mathrm{q}_{\mathrm{ck}}+\mathrm{q}_{\mathrm{re}}+\mathrm{q}_{\mathrm{d}}\right) O(1) \\
+\left(2 \mathrm{q}_{\mathrm{c}}+2 \mathrm{q}_{\mathrm{u}}+6 \mathrm{q}_{\mathrm{rk}}+\mathrm{q}_{\mathrm{ck}}+\left(\mathrm{q}_{\mathrm{re}}+1\right)\left(2 \mathrm{q}_{\mathrm{d}}+\left(2 \mathrm{q}_{\mathrm{H}_{2}}+2 \mathrm{q}_{\mathrm{H}_{3}}\right) \mathrm{q}_{\mathrm{d}}\right)\right) \mathrm{t}_{\mathrm{exp}}+\left(\mathrm{q}_{\mathrm{re}}+\mathrm{q}_{\mathrm{d}}\right) \mathrm{t}_{\mathrm{p}} \\
\varepsilon^{\prime} \geq \frac{1}{\mathrm{q}_{\mathrm{H}_{7}}}\left(\frac{\varepsilon-\psi}{\varepsilon\left(1+\mathrm{q}_{\mathrm{rk}}\right)}+\frac{\varepsilon-\psi}{\varepsilon\left(1+\mathrm{q}_{\mathrm{ck}}\right)}-\frac{\mathrm{q}_{\mathrm{H}_{4}}}{2^{\mathrm{1}_{0} \mathrm{l}_{1}}}-\frac{\left(\mathrm{q}_{\mathrm{H}_{2}} \mathrm{q}_{\mathrm{H}_{3}}+\mathrm{q}_{\mathrm{H}_{3}} \mathrm{q}_{\mathrm{H}_{7}}+\mathrm{q}_{\mathrm{H}_{7}} \mathrm{q}_{\mathrm{H}_{2}}\right)\left(\mathrm{q}_{\mathrm{re}}+\mathrm{q}_{\mathrm{d}}\right)}{4^{1_{0}+\mathrm{l}_{1}}}\right. \\
\left.-\frac{\left(\mathrm{q}_{\mathrm{H}_{2}}+\mathrm{q}_{\mathrm{H}_{3}}+\mathrm{q}_{\mathrm{H}_{7}}\right)\left(\mathrm{q}_{\mathrm{re}}+\mathrm{q}_{\mathrm{d}}\right)}{2^{\mathrm{1}_{0}+\mathrm{l}_{1}-1}}-\frac{3\left(\mathrm{q}_{\mathrm{re}}+\mathrm{q}_{\mathrm{d}}\right)}{\mathrm{q}}\right)
\end{gathered}
$$

where $t_{\exp }$ denotes the running time of an exponentiation in group $G$ and $t_{p}$ denotes the running time of a pairing in groups $\left(G, G_{T}\right)$.

2. or an attacker who breaks the EUF-CMA security of the Schnorr signature with advantage $\psi$ within time $\mathrm{t}^{\prime}$.

PROOF. Without loss of generality, we assume that the Schnorr signature is $\left(\mathrm{t}^{\prime}, \psi\right)$-EUF-CMA secure for some probability $0<\psi<\varepsilon$. If there exists a t-time adversary $\mathcal{A}$ who can break the IND-CPRE-CCA security of our scheme with advantage $\varepsilon-\psi$, then we show how to construct an algorithm $B$ which can break the $\left(\mathrm{t}^{\prime}, \varepsilon^{\prime}\right)-\mathrm{mCBDH}$ assumption in $\mathrm{G}$. Suppose $\mathcal{B}$ is given as input a challenge tuple $\left(\mathrm{g}, \mathrm{g}^{\frac{1}{\mathrm{a}}}, \mathrm{g}^{\mathrm{a}}, \mathrm{g}^{\mathrm{b}}, \mathrm{g}^{\mathrm{c}}\right)$ with unknown a,b.c $\stackrel{\$}{\leftarrow} \mathrm{Z}_{\mathrm{q}}^{*}$. Algorithm $\mathfrak{B}^{\prime} \mathrm{s}$ goal is to output $\hat{\mathrm{e}}(\mathrm{g}, \mathrm{g})^{\mathrm{abc}}$. Algorithm $\mathscr{B}$ first gives (q, G, g, $\mathrm{H}_{1}, \ldots, \mathrm{H}_{7}, l_{0}, l_{1}$ ) to $\mathcal{A}$. Next, $\mathcal{B}$ acts as a challenger and plays the IND-CPRE-CCA game with adversary $\mathcal{A}$ in the following way: 
International Journal of Network Security \& Its Applications (IJNSA), Vol.4, No.2, March 2012

Hash Oracle Queries. At any time adversary $\mathcal{A}$ can issue random oracle queries $\mathrm{H}_{\mathrm{i}}$ with $\mathrm{i} \in\{1$, $\ldots, 7\}$. Algorithm $\mathcal{B}$ maintains seven hash lists $\mathrm{H}_{\mathrm{i}}^{\text {list }}$ with $\mathrm{i} \in\{1, \ldots, 7\}$ which are initially empty, and responds as below:

- $\quad H_{1}$ queries: If $\mathrm{H}_{1}(\mathrm{~h}, \pi)$ has appeared on the $\mathrm{H}_{1}^{\text {list }}$ in a tuple $(\mathrm{h}, \pi, \mathrm{v})$, return the predefined value v. Otherwise, choose $\mathrm{v} \stackrel{\$}{\leftarrow} \mathrm{Z}_{\mathrm{q}}^{*}$ and add tuple $(\mathrm{h}, \pi, \mathrm{v})$ to $\mathrm{H}_{1}^{\text {list }}$ and respond with $\mathrm{H}_{1}(\mathrm{~h}$, $\pi)=\mathrm{v}$.

- $\mathrm{H}_{2}$ queries: If $\mathrm{H}_{2}\left(\mathrm{~m}, \mathrm{r}^{\prime}, \mathrm{pk}_{\mathrm{i}}, \mathrm{w}\right)$ has appeared on the $\mathrm{H}_{2}^{\text {list }}$ in a tuple $\left(\mathrm{m}, \mathrm{r}^{\prime}, \mathrm{pk}, \mathrm{w}, \mathrm{r}\right)$, return the predefined value $r$. Otherwise, choose $r \stackrel{\$}{\leftarrow} Z_{\mathrm{q}}^{*}$ and add tuple $\left(\mathrm{m}, \mathrm{r}^{\prime}, \mathrm{pk}_{\mathrm{i}}, \mathrm{w}, \mathrm{r}\right)$ to $\mathrm{H}_{2}^{\text {list }}$ and respond with $\mathrm{H}_{2}\left(\mathrm{~m}, \mathrm{r}^{\prime}, \mathrm{pk}_{\mathrm{i}}, \mathrm{w}\right)=\mathrm{r}$.

- $H_{3}$ queries: If $\mathrm{H}_{3}(\mathrm{R})$ has appeared on the $\mathrm{H}_{3}^{\text {list }}[\mathrm{R} \in \mathrm{G}]$ in a tuple $(\mathrm{R}, \beta)$, return the predefined value $\beta$. Otherwise, choose $\beta \leftarrow\{0,1\}^{\mathrm{l}_{0}+\mathrm{l}_{1}}$, add tuple $(\mathrm{R}, \beta)$ to $\mathrm{H}_{3}^{\text {list }}$ and respond with $\mathrm{H}_{3}(\mathrm{R})=\beta$.

- $H_{4}$ queries: If $\mathrm{H}_{4}(\mathrm{~A}, \mathrm{~B}, \mathrm{C}, \mathrm{D})$ has appeared on the $\mathrm{H}_{4}^{\text {list }}$ in a tuple $(\mathrm{A}, \mathrm{B}, \mathrm{C}, \mathrm{D}, \gamma)$, return the predefined value $\gamma$. Otherwise, choose $\gamma \stackrel{\$}{\leftarrow} \mathrm{Z}_{\mathrm{q}}^{*}$, add tuple $(\mathrm{A}, \mathrm{B}, \mathrm{C}, \mathrm{D}, \gamma)$ to $\mathrm{H}_{4}^{\text {list }}$ and respond with $\mathrm{H}_{4}(\mathrm{~A}, \mathrm{~B}, \mathrm{C}, \mathrm{D})=\gamma$.

- $H_{5}$ queries: If $\mathrm{H}_{5}(\mathrm{pk})$ has appeared on the $\mathrm{H}_{5}^{\text {list }}$ in a tuple $(\mathrm{pk}, \tau)$, return the predefined value $\tau$. Otherwise, choose $\tau \stackrel{\$}{\leftarrow} Z_{\mathrm{q}}^{*}$, add tuple $(\mathrm{pk}, \tau)$ to $\mathrm{H}_{5}^{\text {list }}$ and respond with $\mathrm{H}_{5}(\mathrm{pk})=\tau$.

- $H_{6}$ queries: If $\mathrm{H}_{6}(\mathrm{w}, \mathrm{pk})$ has appeared on the $\mathrm{H}_{6}^{\text {list }}$ in a tuple (w, pk, $\mathrm{t}, \mathrm{S}$, coin), return the predefined value $S$. Otherwise, choose $\mathrm{t} \stackrel{\$}{\leftarrow} Z_{\mathrm{q}}^{*}$. Next, using the Coron's technique [6], flip a random biased coin [coin $\in\{0,1\}$ ] that yields 0 with a probability $\theta$ and 1 with probability $1-\theta$. If coin $=0$, compute $S=g^{t}$. Otherwise, compute $S=\left(g^{b}\right)^{t}$. Add the tuple (w, pk, t, S, coin) to $\mathrm{H}_{6}^{\text {list }}$ and respond with $\mathrm{H}_{6}(\mathrm{w}, \mathrm{pk})=\mathrm{S}$.

- $H_{7}$ queries: If $\mathrm{H}_{7}(\mathrm{U})$ has appeared on the $\mathrm{H}_{7}^{\text {list }}\left[\mathrm{U} \in \mathrm{G}_{\mathrm{T}}\right]$ in a tuple $(\mathrm{U}, \eta)$, return the predefined value $\eta$. Otherwise, choose $\eta \stackrel{\$}{\leftarrow}\{0,1\}^{\mathrm{l}_{0}+\mathrm{l}_{1}}$, add tuple $(\mathrm{U}, \eta)$ to $\mathrm{H}_{7}^{\text {list }}$ and respond with $\mathrm{H}_{7}(\mathrm{U})=\eta$.

Phase 1. In this phase, adversary $\mathcal{A}$ issues a series of queries subject to the restrictions of the Type II IND-CPRE-CCA game. $\mathscr{B}$ maintains three lists $\mathrm{K}^{\text {list }}, \mathrm{R}^{\text {list }}$ and $\mathrm{C}^{\text {list }}$ which are initially empty, and answers these queries for $\mathcal{A}$ as follows:

- Uncorrupted key generation query. $\mathscr{B}$ picks $x_{i, 1}, x_{i, 2} \stackrel{\$}{\leftarrow} Z_{q}^{*}$. Next, it defines $c_{i}=0$ and $\mathrm{pk}_{\mathrm{i}}=\left(\left(\mathrm{g}^{\frac{1}{\mathrm{a}}}\right)^{\mathrm{x}_{\mathrm{i}, 1}},\left(\mathrm{~g}^{\frac{1}{\mathrm{a}}}\right)^{\mathrm{x}_{\mathrm{i}, 2}}\right)$. Then it adds the tuple $\left(\mathrm{pk}_{\mathrm{i}}, \mathrm{x}_{\mathrm{i}, 1}, \mathrm{x}_{\mathrm{i}, 2}, \mathrm{c}_{\mathrm{i}}\right)$ to $\mathrm{K}^{\text {list }}$ and returns $\mathrm{pk}_{\mathrm{i}}$. Here the bit $c_{i}$ is used to denote whether the secret key with respect to $\mathrm{pk}_{\mathrm{i}}$ is corrupted, i.e., $\mathrm{c}_{\mathrm{i}}=0$ means uncorrupted and $\mathrm{c}_{\mathrm{i}}=1$ means corrupted.

- Corrupted key generation query. $\mathcal{B}$ picks $\mathrm{x}_{\mathrm{i}, 1}, \mathrm{x}_{\mathrm{i}, 2} \stackrel{\$}{\leftarrow} \mathrm{Z}_{\mathrm{q}}^{*}$ and defines $\mathrm{pk}_{\mathrm{i}}=\left(\mathrm{g}^{\mathrm{x}_{\mathrm{i}, 1}}, \mathrm{~g}^{\mathrm{x}_{\mathrm{i}, 2}}\right), \mathrm{c}_{\mathrm{i}}=$ 1. Then, it adds the tuple $\left(\mathrm{pk}_{\mathrm{i}}, \mathrm{x}_{\mathrm{i}, 1}, \mathrm{x}_{\mathrm{i}, 2}, \mathrm{c}_{\mathrm{i}}\right)$ to $\mathrm{K}^{\text {list }}$ and returns $\left(\mathrm{pk}_{\mathrm{i}},\left(\mathrm{x}_{\mathrm{i}, 1}, \mathrm{x}_{\mathrm{i}, 2}\right)\right)$.

- Re-encryption key generation query $\left(\mathrm{pk}_{\mathrm{i}}, \mathrm{pk}_{\mathrm{j}}\right)$. If $\mathrm{R}^{\text {list }}$ has an entry for $\left(\mathrm{pk}_{\mathrm{i}}, \mathrm{pk}_{\mathrm{j}}\right)$, return the predefined re-encryption key to $\mathcal{A}$. Otherwise, algorithm $\mathscr{B}$ acts as follows:

1. Recover tuples $\left(\mathrm{pk}_{\mathrm{i}}, \mathrm{x}_{\mathrm{i}, 1}, \mathrm{x}_{\mathrm{i}, 2}, \mathrm{c}_{\mathrm{i}}\right)$ and $\left(\mathrm{pk}_{\mathrm{j}}, \mathrm{x}_{\mathrm{j}, 1}, \mathrm{x}_{\mathrm{j}, 2}, \mathrm{c}_{\mathrm{j}}\right)$ from $\mathrm{K}^{\text {list }}$. 
International Journal of Network Security \& Its Applications (IJNSA), Vol.4, No.2, March 2012

2. Pick $\mathrm{h} \stackrel{\$}{\leftarrow}\{0,1\}^{\mathrm{l}_{0}}$ and $\pi \stackrel{\$}{\leftarrow}\{0,1\}^{\mathrm{l}_{1}}$; compute $\mathrm{v}=\mathrm{H}_{1}(\mathrm{~h}, \pi), \mathrm{V}=\mathrm{g}^{\mathrm{v}}$ and $\mathrm{W}=\mathrm{H}_{3}\left(\mathrm{pk}_{\mathrm{j}, 2}^{\mathrm{v}}\right)$ $\oplus(\mathrm{h} \| \pi)$.

3. Construct the first component $\mathrm{rk}_{\mathrm{i} \rightarrow \mathrm{j}}^{(1)}$ according to the following cases:

- $\mathrm{c}_{\mathrm{i}}=1$ : Define $\mathrm{rk}_{\mathrm{i} \rightarrow \mathrm{j}}^{(1)}=\frac{\mathrm{h}}{\mathrm{x}_{\mathrm{i}, 1} \mathrm{H}_{5}\left(\mathrm{pk}_{\mathrm{i}, 2}\right)+\mathrm{x}_{\mathrm{i}, 2}}$.

- $\quad\left(c_{i}=0 \wedge c_{j}=0\right)$ : Define $\mathrm{rk}_{\mathrm{i} \rightarrow \mathrm{j}}^{(1)}=\frac{\mathrm{h}}{\mathrm{x}_{\mathrm{i}, 1} \mathrm{H}_{5}\left(\mathrm{pk}_{\mathrm{i}, 2}\right)+\mathrm{x}_{\mathrm{i}, 2}}$. Here define $\mathrm{h}=\mathrm{ah}^{\prime}$ so that $\mathrm{rk}_{\mathrm{i} \rightarrow \mathrm{j}}^{(1)}=\frac{\mathrm{h}^{\prime}}{\frac{\mathrm{x}_{\mathrm{i}, 1} \mathrm{H}_{5}\left(\mathrm{pk}_{\mathrm{i}, 2}\right)+\mathrm{x}_{\mathrm{i}, 2}}{\mathrm{a}}}$ where $\mathrm{h}^{\prime} \in\{0,1\}^{\mathrm{l}_{0}}$.

- $\quad\left(c_{i}=0 \wedge c_{j}=1\right)$ : Output "failure" and abort.

4. If $\mathscr{B}$ does not abort, add $\left(\mathrm{pk}_{\mathrm{i}}, \mathrm{pk}_{\mathrm{j}},\left(\mathrm{rk}_{\mathrm{i} \rightarrow \mathrm{j}}^{(1)}, \mathrm{V}, \mathrm{W}\right), \mathrm{h}\right)$ into list $\mathrm{R}^{\text {list }}$, return $\left(\mathrm{rk}_{\mathrm{i} \rightarrow \mathrm{j}}^{(1)}, \mathrm{V}, \mathrm{W}\right)$

- Condition key query $\left(\mathrm{pk}_{\mathrm{i}}, \mathrm{w}\right)$. If $\mathrm{C}^{\text {list }}$ has an entry for $\left(\mathrm{pk}_{\mathrm{i}}, \mathrm{w}\right)$, return the predefined condition key $\mathrm{ck}_{\mathrm{i}, \mathrm{w}}$ to $\mathcal{A}$. Otherwise algorithm $\mathscr{B}$ acts as follows:

1. Recover tuples $\left(\mathrm{pk}_{\mathrm{i}}, \mathrm{x}_{\mathrm{i}, 1}, \mathrm{x}_{\mathrm{i}, 2}, \mathrm{c}_{\mathrm{i}}\right.$ ) from $\mathrm{K}^{\text {list }}$ and $\left(\mathrm{w}, \mathrm{pk}_{\mathrm{i}}, \mathrm{t}, \mathrm{S}\right.$, coin) from $\mathrm{H}_{6}^{\text {list }}$.

2. It constructs the condition key $\mathrm{ck}_{\mathrm{i}, \mathrm{w}}$ for adversary $\mathcal{A}$ according to the following cases:

- $\mathrm{c}_{\mathrm{i}}=1$ : Algorithm $\mathscr{B}$ responds with $\mathrm{ck}_{\mathrm{i}, \mathrm{w}}=\mathrm{S}^{\frac{1}{\mathrm{x}_{\mathrm{i}, 1}}}$.

- $\quad\left(c_{i}=0 \wedge\right.$ coin $\left.=0\right)$ : Algorithm $B$ responds with $\mathrm{ck}_{\mathrm{i}, \mathrm{w}}=\left(\mathrm{g}^{\mathrm{a}}\right)^{\frac{1}{\mathrm{x}_{\mathrm{i}, 1}}}$ which is same as $\mathrm{S}^{\frac{1}{\mathrm{sk}_{\mathrm{i}, 1}}}$.

- $\quad\left(c_{i}=0 \wedge \operatorname{coin}=1\right)$ : Output "failure" and abort.

3. If $\mathscr{B}$ does not abort, add $\left(\mathrm{pk}_{\mathrm{i}}, \mathrm{w}, \mathrm{ck}_{\mathrm{i}, \mathrm{w}}\right)$ to $\mathrm{C}^{\text {list }}$.

- Re-encryption query $\left(\mathrm{pk}_{\mathrm{i}}, \mathrm{pk}_{\mathrm{j}}, \mathrm{w}, \zeta_{\mathrm{i}}\right)$. Algorithm $\mathcal{B}$ parses $\zeta_{\mathrm{i}}=(\mathrm{A}, \mathrm{B}, \mathrm{C}, \mathrm{D}, \mathrm{E})$. Return $\perp$ if Validity() returns $\perp$. Otherwise it does the following:

1. Recover tuples $\left(\mathrm{pk}_{\mathrm{i}}, \mathrm{x}_{\mathrm{i}, 1}, \mathrm{x}_{\mathrm{i}, 2}, \mathrm{c}_{\mathrm{i}}\right)$ and $\left(\mathrm{pk}_{\mathrm{j}}, \mathrm{x}_{\mathrm{j}, 1}, \mathrm{x}_{\mathrm{j}, 2}, \mathrm{c}_{\mathrm{j}}\right)$ from $\mathrm{K}^{\text {list }}$.

2. If $\left(\mathrm{c}_{\mathrm{i}}=0\right)$ does not hold, issue a condition key generation query $\left(\mathrm{pk}_{\mathrm{i}}, \mathrm{w}\right)$ to obtain $\mathrm{ck}_{\mathrm{i}, \mathrm{w}}$ and a re-encryption key query $\left(\mathrm{pk}_{\mathrm{i}}, \mathrm{pk}_{\mathrm{j}}\right)$ to obtain $\mathrm{rk}_{\mathrm{i}} \rightarrow \mathrm{j}$, and then $\operatorname{ReEncrypt}\left(\mathrm{rk}_{\mathrm{i} \rightarrow \mathrm{j}}, \mathrm{ck}_{\mathrm{i}, \mathrm{w}}, \zeta_{\mathrm{i}}, \mathrm{pk}_{\mathrm{i}}, \mathrm{pk}_{\mathrm{j}}\right)$ to $\mathcal{A}$.

3. Else $\mathfrak{B}$ does the following.

- Pick $\mathrm{h} \stackrel{\$}{\leftarrow}\{0,1\}^{1_{0}}$ and $\pi \stackrel{\$}{\leftarrow}\{0,1\}^{1_{1}}$ and compute $\mathrm{v}=\mathrm{H}_{1}(\mathrm{~h}, \pi)$.

- Compute $\mathrm{V}=\mathrm{g}^{\mathrm{v}}$ and $\mathrm{W}=\mathrm{H}_{3}\left(\mathrm{pk}_{\mathrm{j}, 2}^{\mathrm{v}}\right) \oplus(\mathrm{h} \| \pi)$.

- Since the ciphertext is valid, issue a decryption query $\left(\mathrm{pk}_{\mathrm{i}}, \zeta_{\mathrm{i}}\right)$ and get message $\mathrm{m}$.

- $\quad$ Pick $\mathrm{r}^{\prime} \stackrel{\$}{\leftarrow}\{0,1\}^{\mathrm{l}_{1}}$. Compute $\mathrm{r}=\mathrm{H}_{2}\left(\mathrm{~m}, \mathrm{r}^{\prime}, \mathrm{pk}_{\mathrm{i}}, \mathrm{w}\right)$.

- Compute $\mathrm{A}^{\prime}=\mathrm{g}^{\mathrm{rh}}$ and $\mathrm{C}^{\prime}=\mathrm{H}_{3}\left(\mathrm{~g}^{\mathrm{r}}\right) \oplus\left(\mathrm{m} \| \mathrm{r}^{\prime}\right)$.

- Add tuple $(\mathrm{h}, \pi, \mathrm{v})$ to $\mathrm{H}_{1}^{\text {list }}$ and tuple $\left(\mathrm{m}, \mathrm{r}^{\prime}, \mathrm{pk}_{\mathrm{i}}, \mathrm{w}, \mathrm{r}\right)$ to $\mathrm{H}_{2}^{\text {list }}$, if they are not present in their respective lists.

- $\quad \operatorname{Return}\left(\mathrm{A}^{\prime}, \mathrm{C}^{\prime}, \mathrm{V}, \mathrm{W}\right)$ to $\mathcal{A}$ as the re-encrypted ciphertext.

- Decryption query $\left(\mathrm{pk}_{\mathrm{i}}, \mathrm{w}^{\prime}, \zeta_{\mathrm{i}}\right)$ or $\left.\left(\mathrm{pk}_{\mathrm{i}}, \zeta_{\mathrm{i}}\right)\right\} . \mathscr{B}$ recovers tuple $\left(\mathrm{pk}_{\mathrm{i}}, \mathrm{x}_{\mathrm{i}, 1}, \mathrm{x}_{\mathrm{i}, 2}, \mathrm{c}\right)$ from $\mathrm{K}^{\text {list }}$ and $\left(\mathrm{w}, \mathrm{pk}_{\mathrm{i}}, \mathrm{t}, \mathrm{S}\right.$, coin) from $\mathrm{H}_{6}^{\text {list }}$. If $\mathrm{c}=1$, algorithm $\mathrm{B}$ runs $\left.\operatorname{Decrypt}\left(\left(\mathrm{x}_{\mathrm{i}, 1}, \mathrm{x}_{\mathrm{i}, 2}\right), \zeta_{\mathrm{i}}\right)\right)$ and returns the result to $\mathcal{A}$. Otherwise, algorithm $\mathscr{B}$ works according to the following two cases: 
International Journal of Network Security \& Its Applications (IJNSA), Vol.4, No.2, March 2012

- $\zeta_{\mathrm{i}}$ is an original ciphertext $\zeta_{\mathrm{i}}=(\mathrm{A}, \mathrm{B}, \mathrm{C}, \mathrm{D}, \mathrm{E})$ :

1. Return $\perp$ if Validity() returns $\perp$.

2. Search tuples $\left(\mathrm{m}, \mathrm{r}^{\prime}, \mathrm{pk}, \mathrm{w}, \mathrm{r}\right) \in \mathrm{H}_{2}^{\text {list }},(\mathrm{R}, \beta) \in \mathrm{H}_{3}^{\text {list }}$ and $(\mathrm{U}, \gamma)$ from $\mathrm{H}_{7}^{\text {list }}$ such that $\mathrm{pk}_{\mathrm{i}}=\mathrm{pk}, \mathrm{w}=\mathrm{w}^{\prime}, \beta \oplus\left(\mathrm{m} \| \mathrm{r}^{\prime}\right) \oplus \gamma=\mathrm{C}, \mathrm{g}^{\mathrm{r}}=\mathrm{R}$ and $\left(\mathrm{pk}_{\mathrm{i}, 1}^{\mathrm{H}_{5}\left(\mathrm{pk}_{\mathrm{i}, 2}\right)} \mathrm{pk}_{\mathrm{i}, 2}\right)^{\mathrm{r}}=\mathrm{A}$.

3. If yes, return $\mathrm{m}$ to $\mathcal{A}$. Otherwise, return $\perp$.

- $\zeta_{\mathrm{i}}$ is a re-encrypted ciphertext $\zeta_{\mathrm{I}}=\left(\mathrm{A}^{\prime}, \mathrm{C}^{\prime}, \mathrm{V}, \mathrm{W}\right)$ :

1. Search tuples $\left(\mathrm{m}, \mathrm{r}^{\prime}, \mathrm{pk}, \mathrm{w}, \mathrm{r}\right) \in \mathrm{H}_{2}^{\text {list }},(\mathrm{h}, \pi, \mathrm{v}) \in \mathrm{H}_{1}^{\text {list }},(\mathrm{R}, \beta) \in \mathrm{H}_{3}^{\text {list }}$ and $\left(\mathrm{R}^{\prime}\right.$, $\left.\beta^{\prime}\right) \in \mathrm{H}_{3}^{\text {list }}$ such that $\mathrm{pk}_{\mathrm{i}}=\mathrm{pk}, \mathrm{w}=\mathrm{w}^{\prime}, \mathrm{g}^{\mathrm{rh}}=\mathrm{A}^{\prime}, \mathrm{g}^{\mathrm{r}}=\mathrm{R}, \beta \oplus\left(\mathrm{m} \| \mathrm{r}^{\prime}\right)=\mathrm{C}^{\prime}, \mathrm{g}^{\mathrm{v}}=$ $\mathrm{V}, \beta^{\prime} \oplus(\mathrm{h} \| \pi)=\mathrm{W}$ and $\mathrm{pk}_{\mathrm{i}, 2}^{\mathrm{v}}=\mathrm{R}^{\prime}$.

2. If yes, return $\mathrm{m}$ to $\mathcal{A}$. Otherwise, return $\perp$.

Challenge. When $\mathcal{A}$ decides that Phase 1 is over, it outputs a public key $\mathrm{pk}_{\mathrm{i}^{*}}=\left(\mathrm{pk}_{\mathrm{i}^{*}, 1}, \mathrm{pk}_{\mathrm{i}^{*}, 2}\right)$, a condition $\mathrm{w}^{*}$ and two equal-length messages $\mathrm{m}_{0}, \mathrm{~m}_{1} \in\{0,1\}^{l_{0}+l_{1}}$. Algorithm $\mathcal{B}$ responds as follows:

1. Recover tuple $\left(\mathrm{w}^{*}, \mathrm{pk}_{\mathrm{i}}, \mathrm{t}^{*}, \mathrm{~S}^{*}, \mathrm{coin}^{*}\right)$ from $\mathrm{H}_{6}^{\text {list }}$. If coin $\neq 1, \mathfrak{B}$ outputs "failure" and aborts. Otherwise, $\mathcal{B}$ proceeds to execute the following steps.

2. Pick $\mathrm{u}^{*}, \mathrm{e}^{*} \stackrel{\$}{\leftarrow} \mathrm{Z}_{\mathrm{q}}^{*}$ and compute $\mathrm{B}^{*}=\left(\mathrm{g}^{\mathrm{c}}\right)^{\frac{\mathrm{x}_{\mathrm{i}^{*}, 1}}{\mathrm{t}}}$ and $\mathrm{D}^{*}=\left(\left(\mathrm{B}^{*}\right)^{-1} \mathrm{pk}_{\mathrm{i}^{*}, 1}^{\mathrm{u}^{*}}\right)^{\frac{1}{\mathrm{e}^{*}}}$.

3. Pick $C^{*} \stackrel{\$}{\leftarrow}\{0,1\}^{1_{0}+l_{1}}$ and $\mathrm{r}^{\prime} \stackrel{\$}{\leftarrow}\{0,1\}^{\mathrm{l}_{1}}$.

4. Pick a random bit $\delta \stackrel{\$}{\leftarrow}\{0,1\}$ and compute $\mathrm{r}^{*}=\mathrm{H}_{2}\left(\mathrm{~m}_{\delta}, \mathrm{r}^{\prime}, \mathrm{pk}_{\mathrm{i} *}, \mathrm{w}^{*}\right)$.

5. Compute $\mathrm{A}^{*}=\left(\left(\mathrm{g}^{\frac{1}{\mathrm{a}}}\right)^{\mathrm{x}_{\mathrm{i}^{*}, 1} \mathrm{H}_{5}\left(\mathrm{pk}_{\mathrm{i}^{*}, 2}\right)+\mathrm{x}_{\mathrm{i}^{*}, 2}}\right)^{\mathrm{r}^{*}}$ and $\mathrm{E}^{*}=\mathrm{u}^{*}$.

6. Define $\mathrm{H}_{4}\left(\mathrm{~A}^{*}, \mathrm{~B}^{*}, \mathrm{C}^{*}, \mathrm{D}^{*}\right)=\mathrm{e}^{*}$.

7. Implicitly define $\mathrm{H}_{7}\left(\hat{\mathrm{e}}(\mathrm{g}, \mathrm{g})^{\mathrm{abc}}\right)=\mathrm{C} * \oplus\left(\mathrm{m}_{\delta} \| \mathrm{r}^{\prime}\right) \oplus \mathrm{H}_{3}\left(\mathrm{~g}^{\mathrm{r}}\right)$ (note that $\mathfrak{B}$ does not know $\left.\hat{\mathrm{e}}(\mathrm{g}, \mathrm{g})^{\mathrm{abc}}\right)$.

8. Note that $\hat{\mathrm{e}}(\mathrm{g}, \mathrm{g})^{\mathrm{abc}}=\hat{\mathrm{e}}\left(\mathrm{g}^{\frac{\mathrm{cx} \mathrm{i}^{*}, 1}{\mathrm{t}}}, \mathrm{g}^{\mathrm{bt}}\right)^{\frac{\mathrm{a}}{\mathrm{x}_{\mathrm{i}, 1}}}=\hat{\mathrm{e}}\left(\mathrm{B}^{*}, \mathrm{H}_{6}\left(\mathrm{w}^{*}, \mathrm{pk}_{\mathrm{i}^{*}}\right)\right)^{\frac{1}{\mathrm{sk} \mathrm{i}^{*}}}$.

9. Return $\zeta^{*}=\left(\mathrm{A}^{*}, \mathrm{~B}^{*}, \mathrm{C}^{*}, \mathrm{D}^{*}, \mathrm{E}^{*}\right)$ as the challenged ciphertext to adversary $\mathcal{A}$.

Observe that the challenge ciphertext $\zeta^{*}$ is identically distributed as the real one from the construction. To see this, letting $\mathrm{s}^{*}=\frac{\mathrm{ac}}{\mathrm{t}}$, we have

$$
\begin{aligned}
\mathrm{A}^{*} & =\left(\left(\mathrm{g}^{\frac{1}{\mathrm{a}}}\right)^{\mathrm{x}_{\mathrm{i}^{*}, 1} \mathrm{H}_{5}\left(\mathrm{pk}_{\mathrm{i}^{*}, 2}\right)+\mathrm{x}_{\mathrm{i}^{*}, 2}}\right)^{\mathrm{r}^{*}}=\left(\mathrm{pk}_{\mathrm{i}^{*}, 1}^{\mathrm{H}_{5}\left(\mathrm{pk}_{\mathrm{i}^{*}, 2}\right)} \mathrm{pk}_{\mathrm{i}^{*}, 2}\right)^{\mathrm{r}^{*}} \\
\mathrm{~B}^{*} & =\left(\mathrm{g}^{\mathrm{c}}\right)^{\frac{\mathrm{x}^{*}, 1}{\mathrm{t}}}=\left(\mathrm{g}^{\frac{\mathrm{x}^{*}, 1}{\mathrm{a}}}\right)^{\frac{\mathrm{ac}}{\mathrm{t}}}=\mathrm{pk}_{\mathrm{i}^{*}, 1}^{\mathrm{s}^{*}} \\
\mathrm{C}^{*} & =\mathrm{H}_{3}\left(\mathrm{~g}^{\mathrm{r}^{*}}\right) \oplus\left(\mathrm{m}_{\delta} \| \mathrm{r}^{\prime}\right) \oplus \mathrm{H}_{7}\left(\hat{\mathrm{e}}(\mathrm{g}, \mathrm{g})^{\mathrm{abc}}\right) \\
& =\mathrm{H}_{3}\left(\mathrm{~g}^{\mathrm{r}^{*}}\right) \oplus\left(\mathrm{m}_{\delta} \| \mathrm{r}^{\prime}\right) \oplus \mathrm{H}_{7}\left(\hat{\mathrm{e}}\left(\mathrm{g}, \mathrm{g}^{\mathrm{bt}}\right)^{\frac{\mathrm{ac}}{\mathrm{t}}}\right) \\
& =\mathrm{H}_{3}\left(\mathrm{~g}^{\mathrm{r}^{*}}\right) \oplus\left(\mathrm{m}_{\delta} \| \mathrm{r}^{\prime}\right) \oplus \mathrm{H}_{7}\left(\hat{\mathrm{e}}\left(\mathrm{g}, \mathrm{H}_{6}\left(\mathrm{w}^{*}, \mathrm{pk}_{\mathrm{i}^{*}, \mathrm{w}^{*}}\right)\right)^{\mathrm{s}^{*}}\right) \\
\mathrm{D}^{*} & =\left(\left(\mathrm{B}^{*}\right)^{-1} \mathrm{pk}_{\mathrm{i}^{*}, 1}^{\mathrm{u}^{*}}\right)^{\frac{1}{\mathrm{e}^{*}}} \\
\mathrm{E}^{*} & =\mathrm{u}^{*}
\end{aligned}
$$

Since $\mathrm{u}^{*}$ and $\mathrm{e}^{*}$ are random, adversary cannot distinguish $\mathrm{D}^{*}$ and $\mathrm{E}^{*}$ from the real one. 
International Journal of Network Security \& Its Applications (IJNSA), Vol.4, No.2, March 2012

Phase 2. Adversary $\mathcal{A}$ continues to issue queries as in Phase 1 , with the restrictions prescribed in the IND-CPRE-CCA game. Algorithm $\mathscr{B}$ responds to these queries for $\mathcal{A}$ as in Phase 1.

Guess. Eventually, adversary $\mathcal{A}$ returns a guess $\delta^{\prime} \in\{0,1\}$ to $\mathcal{B}$. Algorithm $\mathscr{B}$ randomly picks a tuple $(\mathrm{U}, \gamma)$ from the $\mathrm{H}_{7}^{\text {list }}$ and outputs $\mathrm{U}$ as the solution to the given problem instance.

Analysis. Now let's analyse the simulation. From the constructions of $\mathrm{H}_{1}, \mathrm{H}_{2}, \mathrm{H}_{3}, \mathrm{H}_{5}$ and $\mathrm{H}_{6}$, it is clear that the simulations of these oracles are perfect. Let $\mathrm{AskH}_{4}^{*}$ be the event that A queried $\left(\mathrm{A}^{*}, \mathrm{~B}^{*}, \mathrm{C}^{*}, \mathrm{D}^{*}\right.$ ) to $\mathrm{H}_{4}$ before challenge phase. The simulation of $\mathrm{H}_{4}$ is perfect as long as $\mathrm{AskH}_{4}^{*}$ did not occur. Since $\mathrm{C}^{*}$ is randomly chosen from $\{0,1\}^{l_{0}+l_{1}}$ by the challenger in the challenge phase, we have $\operatorname{Pr}\left[\mathrm{AskH}_{4}^{*}\right]=\frac{\mathrm{q}_{\mathrm{H}_{4}}}{2^{1_{0}+1_{1}}}$. Let $\operatorname{AskH}_{7}^{*}$ be the event that $\hat{e}(\mathrm{~g}, \mathrm{~g})^{\text {abc }}$ has been queried to $\mathrm{H}_{7}$. The simulation of $\mathrm{H}_{7}$ is perfect as long as $\mathrm{AskH}_{7}^{*}$ did not occur.

$\mathscr{B}$ 's responses to $\mathcal{A}$ 's uncorrupted/corrupted key generation queries are perfect. Let Abort denote the event of $\mathscr{B}$ 's aborting during the simulation of the re-encryption key queries, condition key queries or in the challenge phase. We have $\operatorname{Pr}[\neg$ Abort $\} \geq \theta^{q_{\text {ck }}}(1-\theta)+\theta^{q_{\text {ck }}}(1-\theta)$, which is maximized when each of the two terms are maximized. First term maximizes at $\theta_{\mathrm{opt1}}=\frac{\mathrm{q}_{\mathrm{rk}}}{1+\mathrm{q}_{\mathrm{rk}}}$ and the second term maximizes at $\theta_{\mathrm{opt2}}=\frac{\mathrm{q}_{\mathrm{ck}}}{1+\mathrm{q}_{\mathrm{ck}}}$. Thus the probability $\operatorname{Pr}[\neg$ Abort $]$ is at least $\frac{1}{\mathrm{e}\left(1+\mathrm{q}_{\mathrm{rk}}\right)}+\frac{1}{\mathrm{e}\left(1+\mathrm{q}_{\mathrm{ck}}\right)}$. Here we assume that probability of a key being uncorrupted is same as $\theta$ in $\mathrm{H}_{6}$ queries and $1-\theta$ if it is a corrupted one.

The simulation of the re-encryption key queries is same as the real one, except for the case $\left(c_{i}=\right.$ $0 \wedge c_{j}=0$ ), in which the component $\mathrm{rk}_{\mathrm{i} \rightarrow \mathrm{j}}^{(1)}$ is chosen by choosing $\mathrm{h}$ randomly, where $\mathrm{h}$ is defined as ah'. If Abort does not happen, this is computationally indistinguishable from the real world because :

1. Secret key $\mathrm{sk}_{\mathrm{j}}$ is unknown to $\mathcal{A}$ since $\mathrm{c}_{\mathrm{j}} \neq 1$.

2. $\mathrm{h}$ is encrypted under $\mathrm{pk}_{\mathrm{j}}$ using the "hashed" ElGamal encryption scheme. So, if $\mathcal{A}$ can distinguish $\mathrm{rk}_{\mathrm{i} \rightarrow \mathrm{j}}$ from $\mathrm{rk}_{\mathrm{i} \rightarrow \mathrm{j}}^{\prime}$, it means that $\mathcal{A}$ can determine $(\mathrm{V}, \mathrm{W})$ is an encryption of $\mathrm{h}$ or $\mathrm{h}^{\prime}$, which breaks the CCA security of the "hashed" ElGamal based on the CDH assumption.

The re-encryption queries are also perfect, unless $\mathcal{A}$ can submit valid original ciphertexts without querying $\mathrm{H}_{2}$ or $\mathrm{H}_{3}$ or $\mathrm{H}_{7}$ (denote this event by REErr). This is because we issue a decryption query in the third case of the re-encryption query. We will calculate Pr[REErr] shortly.

The simulation of the decryption oracle is perfect, with the exception that simulation errors may occur in rejecting some valid ciphertexts. A can submit valid original ciphertexts without querying $\mathrm{H}_{2}$ or $\mathrm{H}_{3}$ or $\mathrm{H}_{7}$ (denote this event by DErr). Let Valid be the event that the ciphertext is valid. Let $\mathrm{AskH}_{7}, \mathrm{AskH}_{3}$, and $\mathrm{AskH}_{2}$ be the events $\hat{\mathrm{e}}(\mathrm{g}, \mathrm{g})^{\text {abc }}$ has been queried to $\mathrm{H}_{7}, \mathrm{~g}^{\mathrm{r}}$ has been queried to $\mathrm{H}_{3}$, and $\left(\mathrm{m}, \mathrm{r}^{\prime}, \mathrm{w}\right)$ has been queried to $\mathrm{H}_{2}$ respectively. We have, 
International Journal of Network Security \& Its Applications (IJNSA), Vol.4, No.2, March 2012

$\operatorname{Pr}\left[\right.$ Valid I $\neg \mathrm{AskH}_{2}$ ]

$$
\begin{aligned}
& =\operatorname{Pr}\left[\text { Valid } \wedge \mathrm{AskH}_{3} \wedge \mathrm{AskH}_{7} \mid \neg \mathrm{AskH}_{2}\right]+\operatorname{Pr}\left[\text { Valid } \wedge \mathrm{AskH}_{3} \wedge \neg \mathrm{AskH}_{7} \mid \neg \mathrm{AskH}_{2}\right] \\
& +\operatorname{Pr}\left[\text { Valid } \wedge \neg \mathrm{AskH}_{3} \wedge \text { AskH}_{7} \mid \neg \mathrm{AskH}_{2}\right]+\operatorname{Pr}\left[\text { Valid } \wedge \text { AskH }_{3} \wedge \neg \mathrm{AskH}_{7} \mid \neg \mathrm{AskH}_{2}\right] \\
& =\operatorname{Pr}\left[\text { Valid } \wedge \mathrm{AskH}_{3} \wedge \mathrm{AskH}_{7} \mid \neg \mathrm{AskH}_{2}\right]+\operatorname{Pr}\left[\text { Valid } \wedge \mathrm{AskH}_{3} \mid \neg \mathrm{AskH}_{7} \wedge \neg \mathrm{AskH}_{2}\right] \\
& +\operatorname{Pr}\left[\text { Valid } \wedge \neg \mathrm{AskH}_{7}\left|\neg \mathrm{AskH}_{3}\right| \neg \mathrm{AskH}_{2}\right]+\operatorname{Pr}\left[\text { Valid } \mathrm{I} \neg \mathrm{AskH}_{3} \wedge \neg \mathrm{AskH}_{7} \wedge \neg \mathrm{AskH}_{2}\right] \\
& \leq \frac{\mathrm{q}_{\mathrm{H}_{3}} \mathrm{q}_{\mathrm{H}_{7}}}{4^{1_{0}+1_{1}}}+\frac{\mathrm{q}_{\mathrm{H}_{3}}+\mathrm{q}_{\mathrm{H}_{7}}}{2^{1_{0}+1_{1}}}+\frac{1}{\mathrm{q}}
\end{aligned}
$$

Similarly, we have

$\operatorname{Pr}\left[\right.$ Valid $\left.\mid \neg A_{\text {skH }}\right] \leq \frac{\mathrm{q}_{\mathrm{H}_{2}} \mathrm{q}_{\mathrm{H}_{7}}}{4^{1_{0}+1_{1}}}+\frac{\mathrm{q}_{\mathrm{H}_{2}}+\mathrm{q}_{\mathrm{H}_{7}}}{2^{1_{0}+1_{1}}}+\frac{1}{\mathrm{q}}$ and $\operatorname{Pr}\left[\right.$ Valid $\left.\mid \neg A s k H_{7}\right] \leq \frac{\mathrm{q}_{\mathrm{H}_{2}} \mathrm{q}_{\mathrm{H}_{2}}}{4^{1_{0}+1_{1}}}+\frac{\mathrm{q}_{\mathrm{H}_{2}}+\mathrm{q}_{\mathrm{H}_{3}}}{2^{1_{0}+1_{1}}}+\frac{1}{\mathrm{q}}$

Thus we have,

$$
\begin{aligned}
& \operatorname{Pr}\left[\text { Valid I }\left(\neg \mathrm{AskH}_{2} \vee \neg \mathrm{AskH}_{3} \vee \neg \mathrm{AskH}_{7}\right)\right] \\
& =\operatorname{Pr}\left[\text { Valid } I \neg \text { AskH }_{2}\right]+\operatorname{Pr}\left[\text { Valid } I \neg \text { AskH }_{3}\right]+\operatorname{Pr}\left[\text { Valid } I \neg \text { AskH }_{7}\right] \\
& \leq \frac{\mathrm{q}_{\mathrm{H}_{2}} \mathrm{q}_{\mathrm{H}_{3}}+\mathrm{q}_{\mathrm{H}_{3}} \mathrm{q}_{\mathrm{H}_{7}}+\mathrm{q}_{\mathrm{H}_{7}} \mathrm{q}_{\mathrm{H}_{2}}}{4^{1_{0}+1_{1}}}+\frac{\mathrm{q}_{\mathrm{H}_{2}}+\mathrm{q}_{\mathrm{H}_{3}}+\mathrm{q}_{\mathrm{H}_{7}}}{2^{1_{0}+\mathrm{l}_{1}-1}}+\frac{3}{\mathrm{q}}
\end{aligned}
$$

Let DErr be the event that Validl $\left(\neg \mathrm{AskH}_{2} \vee \neg \mathrm{AskH}_{3} \vee \neg \mathrm{AskH}_{7}\right)$ happens during the entire simulation. Then since $\mathcal{A}$ issues utmost $\mathrm{q}_{\mathrm{d}}$ decryption oracles, we have

$\operatorname{Pr}[D E r r] \leq \frac{\left(\mathrm{q}_{\mathrm{H}_{2}} \mathrm{q}_{\mathrm{H}_{3}}+\mathrm{q}_{\mathrm{H}_{3}} \mathrm{q}_{\mathrm{H}_{7}}+\mathrm{q}_{\mathrm{H}_{7}} \mathrm{q}_{\mathrm{H}_{2}}\right) \mathrm{q}_{\mathrm{d}}}{4^{1_{0}+1_{1}}}+\frac{\left(\mathrm{q}_{\mathrm{H}_{2}}+\mathrm{q}_{\mathrm{H}_{3}}+\mathrm{q}_{\mathrm{H}_{7}}\right) \mathrm{q}_{\mathrm{d}}}{2^{1_{0}+1_{1}-1}}+\frac{3 \mathrm{q}_{\mathrm{d}}}{\mathrm{q}}$

By the definition of REErr as stated above, since $\mathcal{A}$ issues utmost qre re-encryption oracles, we have

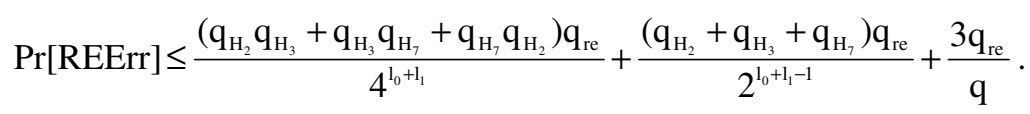

Now, let Good denote the event $\left(\mathrm{AskH}_{7}^{*} \vee \mathrm{AskH}_{4}^{*} \vee \mathrm{REErr} \vee\right.$ DErr $) \mid \neg$ Abort . If Good does not happen, due to the randomness of the output of the random oracle $\mathrm{H}_{7}$, it is clear that $\mathcal{A}$ cannot gain any advantage greater than $\frac{1}{2}$ in guessing $\delta$. Thus we have $\operatorname{Pr}\left[\delta^{\prime}=\delta \mid \neg\right.$ Good $]=\frac{1}{2}$. Hence by splitting $\operatorname{Pr}\left[\delta^{\prime}=\delta\right]$, we have

$$
\begin{aligned}
\operatorname{Pr}\left[\delta^{\prime}=\delta\right]= & \operatorname{Pr}\left[\delta^{\prime}=\delta \mid \neg \text { Good }\right] \operatorname{Pr}[\neg \text { Good }]+\operatorname{Pr}\left[\delta^{\prime}=\delta \mid \text { Good }\right] \operatorname{Pr}[\text { Good }] \\
& \leq \frac{1}{2} \operatorname{Pr}[\neg \text { Good }] \operatorname{Pr}[\text { Good }] \\
& \leq \frac{1}{2}+\frac{1}{2} \operatorname{Pr}[\text { Good }] \\
\operatorname{Pr}\left[\delta^{\prime}=\delta\right] & \geq \operatorname{Pr}\left[\delta^{\prime}=\delta \mid \neg \text { Good }\right] \operatorname{Pr}[\neg \text { Good }] \\
& =\frac{1}{2}-\frac{1}{2} \operatorname{Pr}[\text { Good }]
\end{aligned}
$$

By definition of the advantage for the IND-CPRE-CCA adversary, we then have 
International Journal of Network Security \& Its Applications (IJNSA), Vol.4, No.2, March 2012

$$
\begin{aligned}
& \varepsilon-\psi=\left|2 \times \operatorname{Pr}\left[\delta^{\prime}=\delta\right]-1\right| \\
& \leq \operatorname{Pr}[\text { Good] } \\
& =\operatorname{Pr}\left[\left(\text { AskH }_{7}^{*} \vee \text { AskH }_{4}^{*} \vee \text { REErr } \vee \text { DErr }\right) \mid \neg \text { Abort }\right] \\
& =\frac{\operatorname{Pr}\left[\left(\text { AskH }_{7}^{*} \vee \text { AskH }_{4}^{*} \vee \text { REErr } \vee \text { DErr }\right) \mid \neg \text { Abort }\right]}{\operatorname{Pr}[\neg \text { Abort }]}
\end{aligned}
$$

Substituting values which have been computed, we get

$\operatorname{Pr}\left[\right.$ AskH $\left._{7}^{*}\right] \geq \operatorname{Pr}[\neg$ Abort $] \cdot(\varepsilon-\psi)-\operatorname{Pr}\left[\right.$ AskH$\left._{4}^{*}\right]-\operatorname{Pr}[$ REErr $]-\operatorname{Pr}[$ DErr $]$

$$
\begin{aligned}
\geq & \frac{\epsilon-\psi}{\varepsilon\left(1+\mathrm{q}_{\mathrm{rk}}\right)}+\frac{\epsilon-\psi}{\varepsilon\left(1+\mathrm{q}_{\mathrm{ck}}\right)}-\frac{\mathrm{q}_{\mathrm{H}_{4}}}{2^{1_{0}+1_{1}}}-\frac{\left(\mathrm{q}_{\mathrm{H}_{2}} \mathrm{q}_{\mathrm{H}_{3}}+\mathrm{q}_{\mathrm{H}_{3}} \mathrm{q}_{\mathrm{H}_{7}}+\mathrm{q}_{\mathrm{H}_{7}} \mathrm{q}_{\mathrm{H}_{2}}\right)\left(\mathrm{q}_{\mathrm{re}}+\mathrm{q}_{\mathrm{d}}\right)}{4^{1_{0}+1_{1}}} \\
& -\frac{\left(\mathrm{q}_{\mathrm{H}_{2}}+\mathrm{q}_{\mathrm{H}_{3}}+\mathrm{q}_{\mathrm{H}_{7}}\right)\left(\mathrm{q}_{\mathrm{re}}+\mathrm{q}_{\mathrm{d}}\right)}{2^{\mathrm{1}_{0}+\mathrm{l}_{1}-1}}-\frac{3\left(\mathrm{q}_{\mathrm{re}}+\mathrm{q}_{\mathrm{d}}\right)}{\mathrm{q}}
\end{aligned}
$$

If $\mathrm{AskH}_{7}^{*}$ happens, algorithm $\mathscr{B}$ will be able to solve $\mathrm{mCBDH}$ instance. Therefore we get,

$$
\begin{aligned}
& \mathcal{E}^{\prime} \geq \frac{1}{q_{H_{7}}} \operatorname{Pr}\left[\text { AskH } H_{7}^{*}\right] \\
& \geq \frac{1}{q_{H_{7}}} \frac{\epsilon-\psi}{\varepsilon\left(1+\mathrm{q}_{\mathrm{rk}}\right)}+\frac{\epsilon-\psi}{\varepsilon\left(1+\mathrm{q}_{\mathrm{ck}}\right)}-\frac{\mathrm{q}_{\mathrm{H}_{4}}}{2^{1_{0}+\mathrm{l}_{1}}}-\frac{\left(\mathrm{q}_{\mathrm{H}_{2}} \mathrm{q}_{\mathrm{H}_{3}}+\mathrm{q}_{\mathrm{H}_{3}} \mathrm{q}_{\mathrm{H}_{7}}+\mathrm{q}_{\mathrm{H}_{7}} \mathrm{q}_{\mathrm{H}_{2}}\right)\left(\mathrm{q}_{\mathrm{re}}+\mathrm{q}_{\mathrm{d}}\right)}{4^{1_{0}+\mathrm{l}_{1}}} \\
& \quad-\frac{\left(\mathrm{q}_{\mathrm{H}_{2}}+\mathrm{q}_{\mathrm{H}_{3}}+\mathrm{q}_{\mathrm{H}_{7}}\right)\left(\mathrm{q}_{\mathrm{re}}+\mathrm{q}_{\mathrm{d}}\right)}{2^{\mathrm{1}_{0}+\mathrm{H}_{1}-1}}-\frac{3\left(\mathrm{q}_{\mathrm{re}}+\mathrm{q}_{\mathrm{d}}\right)}{\mathrm{q}}
\end{aligned}
$$

From the description of the simulation, $\mathscr{B}$ 's running time can be bounded by

$$
\begin{aligned}
\mathrm{t}^{\prime} \leq \mathrm{t} & +\left(\mathrm{q}_{\mathrm{H}_{1}}+\mathrm{q}_{\mathrm{H}_{2}}+\mathrm{q}_{\mathrm{H}_{3}}+\mathrm{q}_{\mathrm{H}_{4}}+\mathrm{q}_{\mathrm{H}_{5}}+\mathrm{q}_{\mathrm{H}_{6}}+\mathrm{q}_{\mathrm{H}_{7}}+\mathrm{q}_{\mathrm{u}}+\mathrm{q}_{\mathrm{c}}+\mathrm{q}_{\mathrm{rk}}+\mathrm{q}_{\mathrm{ck}}+\mathrm{q}_{\mathrm{re}}+\mathrm{q}_{\mathrm{d}}\right) O(1) \\
& +\left(2 \mathrm{q}_{\mathrm{c}}+2 \mathrm{q}_{\mathrm{u}}+6 \mathrm{q}_{\mathrm{rk}}+\mathrm{q}_{\mathrm{ck}}+\left(\mathrm{q}_{\mathrm{re}}+1\right)\left(2 \mathrm{q}_{\mathrm{d}}+\left(2 \mathrm{q}_{\mathrm{H}_{2}}+2 \mathrm{q}_{\mathrm{H}_{3}}\right) \mathrm{q}_{\mathrm{d}}\right)\right) \mathrm{t}_{\text {exp }}+\left(\mathrm{q}_{\mathrm{re}}+\mathrm{q}_{\mathrm{d}}\right) \mathrm{t}_{\mathrm{p}}
\end{aligned}
$$

This completes the proof of Theorem 2.

\section{CONCLUSION}

In this paper, we proposed a more efficient CCA secure unidirectional C-PRE scheme with less number of bilinear pairings. The scheme is more elegant when compared to its counterparts. We have proved the security of the scheme in the random oracle model under appropriate security definitions. There are still many open problems to be solved, such as designing CCA secure CPRE scheme in the standard model, C-PRE in other settings like identity based and certificateless cryptography.

\section{REFERENCES}

[1] Ran Canetti and Susan Hohenberger. Chosen-ciphertext secure proxy re-encryption. In ACM Conference on Computer and Communications Security 2007, pages 185-194, 2007.

[2] Jun Shao and Zhenfu Cao. CCA-Secure Proxy Re-encryption without Pairings. In Public Key Cryptography 2009, volume 5443 of LNCS, pages 357-376, 2009.

[3] G. Ateniese, K. Fu, M. Green, and S. Hohenberger. Improved proxy re-encryption schemes with applications to secure distributed storage. In Internet Society (ISOC): NDSS 2005, pages 29-43, 2005.

[4] H. Khurana and R. Koleva. Scalable security and accounting services for content-based publish subscribe systems. International Journal of E-Business Research, 2006. 
International Journal of Network Security \& Its Applications (IJNSA), Vol.4, No.2, March 2012

[5] G. Ateniese, K. Fu, M. Green, and S. Hohenberger. Improved proxy re-encryption schemes with applications to secure distributed storage. ACM Transactions on Information and System Security (TISSEC), pages 1-30, 2006.

[6] Jean-Sebastien Coron. On the Exact Security of Full Domain Hash. In CRYPTO, volume 1880 of LNCS, pages 229-235, 2000.

[7] T.S. Heydt-Benjamin, H. Chae, B. Defend, and K. Fu. Privacy for public transportation. In PET 2006, volume 4258 of LNCS, pages 1-19, 2005.

[8] Cheng-Kang Chu, Jian Weng, Sherman S. M. Chow, Jianying Zhou, and Robert H. Deng. Conditional Proxy Broadcast Re-Encryption. In ACISP 2009, volume 5594 of LNCS, pages 327-342, 2009.

[9] Matt Blaze, Gerrit Bleumer, and Martin Strauss. Divertible protocols and atomic proxy cryptography. In EUROCRYPT 1988, volume 1403 of LNCS, pages 127-144, 1998.

[10] Claus-Peter Schnorr. Efficient Identification and Signatures for Smart Cards. In CRYPTO 1989, volume 435 of LNCS, pages 239-252, 1989.

[11] A. Talmy and O. Dobzinski. Abuse freedom in access control schemes. In AINA 2006, pages 77-86, 2006.

[12] Jian Weng, Sherman S.M. Chow, Yanjiang Yang, and Robert H. Deng. Efficient Unidirectional Proxy Re-Encryption. Cryptology ePrint Archive, Report 2009/189 (2009) http://eprint.iacr.org/.

[13] A. Ivan and Y. Dodis. Proxy cryptography revisited. In Internet Society (ISOC): NDSS 2003, 2003.

[14] Jian Weng, Robert H. Deng, Xuhua Ding, Cheng-Kang Chu, and Junzuo Lai. Conditional proxy re-encryption secure against chosen-ciphertext attack. In ASIACCS, pages 322-332, 2009.

[15] Masahiro Mambo and Eiji Okamoto. Proxy Cryptosystems: Delegation of the Power to Decrypt Ciphertexts. IEICE Trans. Fund. Elect. Communications and CS, E80-A/1:54-63, 1997.

[16] S. Hohenberger, G.N. Rothblum, A. Shelat, and V. Vaikuntanathan. Securely obfuscating reencryption. In TCC 2007, volume 4392 of LNCS, pages 233-252, 2007.

[17] Jian Weng, Yanjiang Yang, Qiang Tang, Robert H. Deng, and Feng Bao. Efficient Conditional Proxy Re-encryption with Chosen-Ciphertext Security. In ISC 2009, volume 5735 of LNCS, pages 151-166, 2009.

[18] Matthew Green and Giuseppe Ateniese. Identity-Based Proxy Re-encryption. In ACNS 2007, volume 4521 of LNCS, pages 288-306, 2007.

[19] Y-P. Chiu, C-L. Lei, and C-Y. Huang. Secure multicast using proxy encryption. In ICICS 2005, volume 3783 of LNCS, pages 280-290, 2005.

[20] H. Khurana and H-S. Hahm. Certified mailing lists. In ASIACCS 2006, pages 46-58, 2006.

[21] G. Taban, A.A. C'ardenas, and V.D. Gligor. Towards a secure and interoperable drm architecture. In ACM DRM 2006, pages 69-78, 2006.

[22] Smith. Tony. Dvd jon: buy drm-less tracks from apple itunes. 2005. http://www.theregister.co.uk/2005/03/18/itunes pymusique.

[23] H. Khurana, A. Slagell, and R. Bonilla. Sels: A secure e-mail list service. In ACM SAC 2005, pages 306-313, 2005.

\section{Authors}

\section{S.Sree Vivek}

$\mathrm{He}$ is currently a $\mathrm{PhD}$ scholar in the Department of Computer Science and Engineering, of IIT Madras. His research focuses on Provably Secure Public Key Cryptosystems.

\section{S.Sharmila Deva Selvi}

She is currently a PhD scholar in the Department of Computer Science and Engineering, of IIT Madras. Her research focuses on Provably Secure Public Key Cryptosystems.
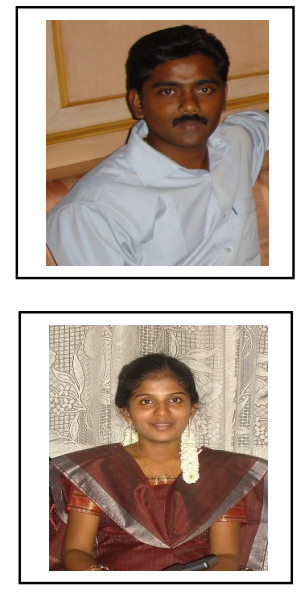
International Journal of Network Security \& Its Applications (IJNSA), Vol.4, No.2, March 2012

\section{Radhakishan}

He is currently pursuing a Bachelor of Technology in the Department of Computer Science and Engineering, of National Institute of Technology, Tiruchirappalli. His research interests include 1) Public key cryptography 2) Randomized Algorithms and 3) Graph theory.

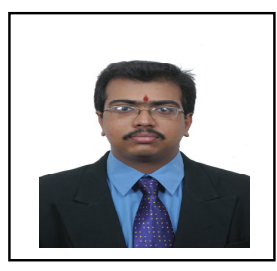

\section{Pandu Rangan}

$\mathrm{He}$ is currently a Professor in the Department of Computer Science and Engineering of IIT Madras. His research focuses on the design of pragmatic algorithms. His research interests include 1) Restricting the problem domain 2) Approximate algorithm design 3) Randomized algorithms 4)Parallel and VLSI algorithms and 5) Cryptography Applications. He is also a Fellow of Indian National Academy of Engineering (FNAE).

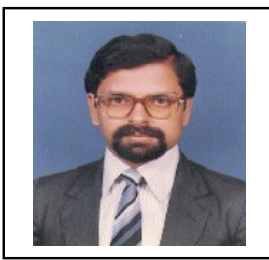

